\title{
Model Building and Optimization Analysis of MDF Continuous Hot-Pressing Process by Neural Network
}

\author{
Qingfa Li, ${ }^{1}$ Yaqiu Liu, ${ }^{1}$ and Liangkuan $\mathrm{Zhu}^{2}$ \\ ${ }^{1}$ School of Information and Computer Engineering, Northeast Forestry University, Harbin 150040, China \\ ${ }^{2}$ College of Electromechanical Engineering, Northeast Forestry University, Harbin, China \\ Correspondence should be addressed to Yaqiu Liu; yaqiuliu@gmail.com
}

Received 8 March 2016; Accepted 9 August 2016

Academic Editor: Yakov Strelniker

Copyright (c) 2016 Qingfa Li et al. This is an open access article distributed under the Creative Commons Attribution License, which permits unrestricted use, distribution, and reproduction in any medium, provided the original work is properly cited.

\begin{abstract}
We propose a one-layer neural network for solving a class of constrained optimization problems, which is brought forward from the MDF continuous hot-pressing process. The objective function of the optimization problem is the sum of a nonsmooth convex function and a smooth nonconvex pseudoconvex function, and the feasible set consists of two parts, one is a closed convex subset of $R^{n}$, and the other is defined by a class of smooth convex functions. By the theories of smoothing techniques, projection, penalty function, and regularization term, the proposed network is modeled by a differential equation, which can be implemented easily. Without any other condition, we prove the global existence of the solutions of the proposed neural network with any initial point in the closed convex subset. We show that any accumulation point of the solutions of the proposed neural network is not only a feasible point, but also an optimal solution of the considered optimization problem though the objective function is not convex. Numerical experiments on the MDF hot-pressing process including the model building and parameter optimization are tested based on the real data set, which indicate the good performance of the proposed neural network in applications.
\end{abstract}

\section{Introduction}

Medium density fibreboard (MDF) finds many applications in wood industries because of its favorable properties such as surface characteristics, dimensional stability, and excellent machinability $[1,2]$. In the MDF hot-pressing process many physical processes are involved and the complexity of this operation arises from the fact that they are coupled. Hot-pressing process is one of the key procedures in the production of MDF, which influences the utilization ratio of energy and resource. With the decreased resource of timber and the increased demand of MDF, it is of great important to analyze the experimental data effectively and reasonably, find the main factors among the many indexes of MDF, and establish the relation models on the properties of slab, the parameters in the hot-pressing process, and the main indexes of MDF. These relation models not only can help the staff give reasonable prediction and a reliability assessment to the hot-pressing process according to the actual process parameters, but also provide a theoretical basis for the setting and adjusting of the main factors in hot-pressing process according to the actual demand of MDF properties. So optimization models and methods have been important tools for the optimization, control, and scheduling of the hotpressing of plates.

Real-time online solutions of optimization problems are desired in many engineering and scientific applications. One possible and very promising approach to solve the real-time optimization problems is to apply artificial neural networks [3-5]. With the resemblance brains, neural networks can be implemented online by hardware and have become an important technical toll for solving optimization problems, for example, [3, 4, 6-9]. Based on the gradient method, the Hopfield neural networks proposed in $[4,5]$ are the two classical recurrent neural networks for linear and nonlinear programming, whereafter, in addition to the gradient method, many types of neural networks are designed, such as the Lagrangian neural networks [10], the projectiontype neural networks [11, 12], the dual network [13], and the stochastic neural network [14]. Projection method is an effective and simple method for solving the constraints. However, it is impossible to solve the general constraints by 
projection method. Then, Lagrangian and penalty methods are introduced into networks. Based on the Lagrangian function method, Lagrangian networks were proposed for solving the optimization problems $[8,10]$ with general constraints. But the Lagrangian network increases the dimension of the networks along with the number of the constraints. In recent years, recurrent neural networks based on penalty method were widely investigated for solving optimization problems. The neural networks for smooth optimization problems can not solve nonsmooth optimization problems, because the gradients of the objective and constrained functions are required in such neural networks. The generalized nonlinear programming circuit (G-NPC) in [15] can be considered as a natural extension of nonlinear programming circuit (NPC) for solving nonsmooth convex optimization problems with inequality constraints. But the nonempty interior of feasible region and large enough penalty parameters are needed for the network in [15]. In order to overcome the nonempty assumption of the interior of feasible region, Bian and Xue [6] proposed a recurrent neural network for nonsmooth convex optimization based on penalty function method. The efficiency of the neural networks for solving convex optimization problems relies on the convexity of functions. A neural network for nonconvex quadratic optimization is presented in [16]. Some neural networks modeled by differential inclusion were also proposed for some nonsmooth and nonconvex optimization problems $[6,17]$. To overcome the differential inclusion, smoothing techniques are introduced into the neural networks. The main feature of smoothing method is to approximate the nonsmooth functions by a class of smooth functions. Thus, the neural network constructed by the smoothing techniques is modeled by a differential equation, which can be implemented easily in circuits and mathematical software [18].

In this paper, we propose a neural network model for solving the optimization problem brought forward from the MDF continuous hot-pressing automatic control system. In Section 2, some notations and necessary preliminary results are listed. In Section 3, based on the SVM theory with the existing linear and nonlinear kernel functions, we give an optimization problem, which includes the problems for building the models of MDF continuous hot-pressing system and optimizing the MDF performance indexes as special cases. In order to build up the relation models on the properties of the slab, technical parameters in hot-pressing process, and the performance indexes of MDF, when the kernel function is positive definite or semipositive definite, the corresponding optimization problem is a constrained convex problem; otherwise it is a nonconvex problem. The optimization problem for optimizing the performance parameters is a nonconvex constrained optimization problem, but its objective function is pseudoconvex due to the appropriate choice of kernel functions. In Section 4, we propose a neural network based on the penalty function method, projection method, and smoothing techniques. The proposed network is modeled by a nonautomatic differential equation. By Lyapunov method, we prove that the solution of the proposed network is global existent and convergent to the feasible set of the considered optimization problems. Moreover, due to the pseudoconvexity of the objective function and the convexity of the constraint, the proposed network also converges to the optimal solution set of the optimization problem. In Section 5, based on the existing data set, we use the proposed network into the model building and parameter optimizing problems of hot-pressing system, which validates the good performance of the obtained results in this paper.

Notations. $R_{+}=[0,+\infty)$. Given column vectors $x=\left(x_{1}\right.$, $\left.x_{2}, \ldots, x_{n}\right)^{T}$ and $y=\left(y_{1}, y_{2}, \ldots, y_{n}\right)^{T},\langle x, y\rangle=x^{T} y=$ $\sum_{i=1}^{n} x_{i} y_{i}$ is the scalar product of $x$ and $y . x_{i}$ denotes the $i$ th element of $x .\|x\|$ denotes the Euclidean 2-norm defined by $\|x\|=\left(\sum_{i=1}^{n} x_{i}^{2}\right)^{1 / 2}$. For a closed convex subset $\Gamma \subseteq R^{n}$, $\operatorname{dist}(x, \Gamma)$ is the distance from $x$ to $\Gamma$ defined by $\operatorname{dist}(x, \Gamma)=$ $\min _{y \in \Gamma}\|x-y\|$.

\section{Preliminaries}

In this section, we state some definitions and properties needed in this paper. We refer the readers to [19-21].

2.1. Support Vector Regression. Kernels were regarded as a function with the formulation of inner product and have been a powerful tool in machine learning for their superior performance over a wide range of learning problems, such as isolated hand written digit recognition, text categorization, and face detection $[21,22]$.

Let $\mathscr{X}$ be a nonempty set and $K: \mathscr{X} \times \mathscr{X} \rightarrow R$ be a realvalued and symmetric function. With the kernel matrix $\mathbf{K}=$ $\left(k\left(x_{i}, x_{j}\right)\right)_{i, j=1}^{n}, K$ is said to be a positive semidefinite kernel, if $\mathbf{K}$ is positive semidefinite for any $n \in \mathbb{N}$ and $x_{1}, x_{2}, \ldots, x_{n} \in$ $\mathscr{X}$. We call $K$ an indefinite kernel, if there exist $x_{1}, x_{2}, \ldots, x_{n} \in$ $\mathscr{X}$ and $v, w \in R^{n}$ such that $v^{T} \mathbf{K} v<0$ and $w^{T} \mathbf{K} w>0$.

In what follows, we list some widely used kernels.

(i) Gaussian radial basis function kernel: $K(x, y)=$ $\exp \left(-\|x-y\|^{2} / 2 \sigma^{2}\right)$

(ii) Polynomial kernel: $K(x, y)=(\langle x, y\rangle+c)^{p}, c \geq 0$, $p \geq 1$.

(iii) Sigmoid kernel: $K(x, y)=\tanh (\kappa\langle x, y\rangle+v), \kappa>0$, $v<0$.

2.2. Smoothing Approximation. Smoothing approximation is an effective method for solving nonsmooth optimization problems and has been widely used in the past decades. The main feature of smoothing method is to approximate the nonsmooth functions by a class of parameterized smooth functions. In this paper, we adopt the smoothing function defined as follows.

Definition 1 (see [23]). Let $g: R^{n} \rightarrow R$ be a continuous function. One calls $\widetilde{g}: R^{n} \times R_{+} \rightarrow R$ a smoothing function of $g$, if $\widetilde{g}(\cdot, \mu)$ is continuously differentiable for any fixed $\mu>0$ and $\lim _{z \rightarrow x, \mu \downarrow 0} \tilde{g}(z, \mu)=g(x)$ holds for any $x \in R^{n}$. 
Chen and Mangasarian constructed a class of smooth approximations of the function $(s)_{+}:=\max \{0, s\}$ by convolution $[20,24]$ as follows. Let $\rho: R \rightarrow R_{+}$be a piecewise continuous density function satisfying

$$
\begin{aligned}
\rho(t) & =\rho(-t), \\
\kappa & :=\int_{-\infty}^{\infty}|t| \rho(t) d t<\infty .
\end{aligned}
$$

Then

$$
\phi(t, \mu):=\int_{-\infty}^{\infty}(s-\mu t)_{+} \rho(t) d t
$$

from $R \times R_{+}$to $R_{+}$is well defined.

By different density functions, many popular smoothing functions of $(s)_{+}$can be derived, such as

$$
\begin{aligned}
& \phi_{1}(s, \mu)=s+\mu \ln \left(1+e^{-s / \mu}\right), \\
& \phi_{2}(s, \mu)=\frac{1}{2}\left(s+\sqrt{s^{2}+4 \mu^{2}}\right) \\
& \phi_{3}(s, \mu)= \begin{cases}\max \{0, s\} & \text { if }|s|>\mu \\
\frac{(s+\mu)^{2}}{4 \mu} & \text { if }|s| \leq \mu,\end{cases} \\
& \phi_{4}(s, \mu)= \begin{cases}s+\frac{\mu}{2} e^{-s / \mu} & \text { if } s>0 \\
\frac{\mu}{2} e^{-s / \mu} & \text { if } s \leq 0,\end{cases}
\end{aligned}
$$

where $\phi_{1}$ is the neural networks smoothing function, $\phi_{2}$ is called the CHKS (Chen-Harker-Kanzow-Smale) smoothing function, $\phi_{3}$ is called the uniform smoothing function, and $\phi_{4}$ is called the Picard smoothing function. The four functions belong to the class of the Chen-Mangasarian smoothing functions.

Many nonsmooth functions can be reformulated by using the plus function. We list some of them as follows:

$$
\begin{aligned}
|s| & =(s)_{+}+(-s)_{+}, \\
\max (s, t) & =s+(t-s)_{+} \\
\min (s, t) & =s-(s-t)_{+}, \\
\operatorname{mid}(s, \ell, u) & =\min (\max (\ell, s), u), \quad \text { for given } \ell, u .
\end{aligned}
$$

So we can define a smoothing function for the above nonsmooth functions by a smoothing function of $(s)_{+}$.

From Theorem 9.61 and Corollary 8.47(b) in [20], when $g: R^{n} \rightarrow R$ is locally Lipschitz continuous at $x$, the subdifferential associated with a smoothing function

$$
\begin{aligned}
& G_{\tilde{g}}(x)=\operatorname{con}\left\{v \mid \nabla_{x} \tilde{g}\left(x^{k}, \mu_{k}\right) \longrightarrow v \text {, for } x^{k} \longrightarrow x, \mu_{k}\right. \\
& \quad \downarrow 0\},
\end{aligned}
$$

is nonempty and bounded, and $\partial g(x) \subseteq G_{\tilde{g}}(x)$, where "con" denotes the convex hull. In [20,23], it is shown that many smoothing functions satisfy the gradient consistency

$$
\partial g(x)=G_{\tilde{g}}(x),
$$

which is an important property of the smoothing methods and guarantees the convergence of smoothing methods with adaptive updating schemes of smoothing parameters to a stationary point of the original problem.

2.3. Pseudoconvex Function. Pseudoconvex function is a class of functions, which may be nonsmooth or nonconvex, but brings us the opportunity to find the optimal solutions.

Definition 2 (see [25]). Let $\mathscr{X}$ be a nonempty convex subset of $R^{n}$. A function $\phi$ is said to be pseudoconvex on $\mathscr{X}$ if, for any $x, y \in \mathscr{X}$, one has

$$
\begin{array}{ll} 
& \exists \xi(x) \in \partial \phi(x) \\
\text { s.t. } & \langle\xi(x), y-x\rangle \geq 0 \Longrightarrow \\
& \phi(y) \geq \phi(x) .
\end{array}
$$

Many nonconvex functions in application are pseudoconvex, such as the Butterworth filter function, fraction function, and density function. Of particular interest in this paper is the fact that the Gaussian function

$$
h(x)=-\exp \left(-\sum_{i=1}^{n} \frac{x_{i}^{2}}{\sigma_{i}^{2}}\right)
$$

with $\sigma_{i}>0, i=1,2, \ldots, n$, is pseudoconvex on $R^{n}$.

2.4. Project Operator. Let $\mathscr{X}$ be a closed convex subset of $R^{n}$. Then the projection operator to $\mathscr{X}$ at $x$ is defined by

$$
P_{X}(x)=\arg \min _{u \in \mathscr{X}}\|u-x\|^{2}
$$

and satisfies the following inequalities:

$$
\begin{aligned}
\left\langle v-P_{\mathscr{X}}(v), P_{\mathscr{X}}(v)-u\right\rangle & \geq 0, \quad \forall v \in R^{n}, u \in \mathscr{X} ; \\
\left\|P_{\mathscr{X}}(u)-P_{\mathscr{X}}(v)\right\| & \leq\|u-v\|, \quad \forall u, v \in R^{n} .
\end{aligned}
$$

(i) Suppose $\Omega=\left\{x \in R^{n}: u \leq x \leq v\right\}$ with $u, v \in R^{n} \cup\{ \pm \infty\}$; then $P_{\Omega}(x)=\left(p_{1}, p_{2}, \ldots, p_{n}\right)^{T}$ can be expressed by

$$
p_{i}= \begin{cases}u_{i} & x_{i}<u_{i} \\ x_{i} & u_{i} \leq x_{i} \leq v_{i} \\ v_{i} & v_{i}<x_{i} .\end{cases}
$$

(ii) Suppose $\Omega=\{x: A x=b\}$ with $A \in R^{r \times n}$ of full row rank and $b \in R^{r}$; then $P_{\Omega}(x)=x-A^{T}\left(A A^{T}\right)^{-1}(A x-b)$.

\section{Optimization Problems in MDF Continuous Hot-Pressing Process}

In this section, we will give the optimization model considered in this paper. First, by the optimization and support vector machine theories, we show the optimization models for building up the relationships in MDF continuous hotpressing process. Then, another optimization model for 
optimizing the parameters in the MDF continuous hotpressing process is obtained. Thus, we express these two kinds of problems into a uniform formulation, which is the optimization problem considered in Section 3.

3.1. Optimization Problem for Building up the Models of MDF Continuous Hot-Pressing Process. Denote $\mathscr{X} \subset R^{p}$ and $\mathcal{Y} \subset R$ as two sets, where $x^{i}=\left(x_{1}^{i}, x_{2}^{i}, \ldots, x_{p}^{i}\right) \in \mathscr{X}$ is the attribute vector on behalf of the hot-pressing plate properties; $y^{i} \in \mathcal{Y}$ indicates the values of the qualities of hot-pressing plate. Let $S=\left(s^{1}, s^{2}, \ldots, s^{n}\right)$ be the training data set of hot-pressing process, where $s^{i}=\left(x^{i}, y^{i}\right)$ obeys the unknown distribution and is IID (independent and identically distributed). Based on the support vector machine theory and the training data set, we would like to find a nonlinear function $y=f(x)$ such that it approximates the training data set as much as possible.

With the kernel function $K$ and the Huber loss function, from the theory in [21], the dual optimization problem of the primal optimization problem of SVM is given as

$$
\begin{array}{ll}
\min _{\alpha, \beta} & \frac{1}{2} \sum_{i, j=1}^{n}\left(\beta_{i}-\alpha_{i}\right)\left(\beta_{j}-\alpha_{j}\right) K\left(x^{i}, x^{j}\right) \\
& -\sum_{i=1}^{n}\left(\beta_{i}-\alpha_{i}\right) y^{i}+\frac{\varepsilon}{2 C} \sum_{i=1}^{n}\left(\beta_{i}-\alpha_{i}\right)^{2} \\
\text { s.t. } \quad \sum_{i=1}^{n}\left(\beta_{i}-\alpha_{i}\right)=0, & 0 \leq \beta_{i}, \alpha_{i} \leq C, \quad i=1,2, \ldots, n .
\end{array}
$$

Denote $\alpha_{i}^{*}$ and $\beta_{i}^{*}$ as the optimal solutions of (12). Then the regression function $f$ can be expressed by

$$
f(x)=\sum_{i=1}^{n}\left(\beta_{i}^{*}-\alpha_{i}^{*}\right) K\left(x^{i}, x\right)+b,
$$

where $b$ can be calculated by one of the following two methods:

$$
\begin{aligned}
& b=y^{j}-\sum_{i=1}^{n}\left(\beta_{i}^{*}-\alpha_{i}^{*}\right)\left\langle x^{i}, x^{j}\right\rangle+\varepsilon, \quad \alpha_{j}^{*} \in(0, C) \\
& b=y^{j}-\sum_{i=1}^{n}\left(\beta_{i}^{*}-\alpha_{i}^{*}\right)\left\langle x^{i}, x^{j}\right\rangle-\varepsilon, \quad \alpha_{j}^{*} \in(0, C) .
\end{aligned}
$$

Denote $x_{i}=\beta_{i}-\alpha_{i}$ in (12); then (12) can be reformulated as

$$
\begin{array}{ll}
\min & \frac{1}{2} x^{T} Q x-y^{T} x \\
\text { s.t. } & e^{T} x=0, \quad-C e \leq x \leq C e,
\end{array}
$$

where $x=\left(x_{1}, x_{2}, \ldots, x_{n}\right)^{T}, y=\left(y^{1}, y^{2}, \ldots, y^{n}\right)^{T}, e=(1,1$, $\ldots, 1)^{T} \in R^{n}$, and $Q=\mathbf{K}+(\varepsilon / 2 C) I_{n}$ with $\mathbf{K}=\left(K\left(x^{i}, x^{j}\right)\right)_{i, j=1}^{n}$. For the optimal solution $x^{*}=\left(x_{1}^{*}, x_{2}^{*}, \ldots, x_{n}^{*}\right)$ of (15), we call $x^{i}$ a support vector if $x_{i}^{*} \neq 0$. The optimal solution of (15) solved by the original methods often has almost $100 \%$ support vectors, which increases the complexity of the relation models largely. Thus, we introduce the problem deduced by (15); that is,

$$
\begin{array}{ll}
\min & \frac{1}{2} x^{T} Q x-y^{T} x+\gamma \sum_{i=1}^{n}\left|x_{i}\right| \\
\text { s.t. } & e^{T} x=0, \quad-C e \leq x \leq C e
\end{array}
$$

with $\gamma>0$. In (43), $\gamma \sum_{i=1}^{n}\left|x_{i}\right|$ is often called the regulation term, which is used to control the number of its support vectors.

Define the penalty function

$$
P(x)=\left\|\frac{1}{n} e e^{T} x\right\|=\frac{\sqrt{n}}{n}\left|\sum_{i=1}^{n} x_{i}\right|,
$$

and then $\{x: P(x) \leq 0\}=\left\{x: e^{T} x=0\right\}$. From [19, Proposition 2.4.3], $x$ is an optimal solution of (43) if and only if it is an optimal solution of the following problem:

$$
\begin{aligned}
& \min \quad \frac{1}{2} x^{T} \mathrm{Q} x-y^{T} x+\gamma \sum_{i=1}^{n}\left|x_{i}\right|+\sigma\left|e^{T} x\right| \\
& \text { s.t. } \quad-C e \leq x \leq C e,
\end{aligned}
$$

where $\sigma=C\|Q\|+\|y\| / \sqrt{n}+\gamma+1$. Thus, we can build up the relation models of hot-pressing process by solving problem (18).

3.2. Optimization Problem for Optimizing the Parameters in MDF Hot-Pressing Process. Based on the relationships built up in Section 3.1, we focus on the modulus of rupture (MOR), modulus of elasticity (MOE), and internal bonding strength (IBS) of hot-pressing plate by optimizing the process parameters and slab attributes. Suppose the regression functions of MOR, MOS, and IBS with respect to some relative parameters based on the Gaussian radial basis function kernel are

$$
\begin{aligned}
& f_{1}(x):=\sum_{i=1}^{n} \rho_{i}^{*} \exp \left(-\frac{\left\|z_{i}-x\right\|^{2}}{2 \sigma^{2}}\right)+b_{1} \\
& f_{2}(x):=\sum_{i=1}^{n} \theta_{i}^{*} \exp \left(-\frac{\left\|z_{i}-x\right\|^{2}}{2 \sigma^{2}}\right)+b_{2} \\
& f_{3}(x):=\sum_{i=1}^{n} \kappa_{i}^{*} \exp \left(-\frac{\left\|z_{i}-x\right\|^{2}}{2 \sigma^{2}}\right)+b_{3}
\end{aligned}
$$

and based on the linear polynomial kernel are

$$
\begin{aligned}
& f_{1}(x):=\sum_{i=1}^{n} \bar{\rho}_{i}^{*}\left\langle z_{i}, x\right\rangle+\bar{b}_{1} \\
& f_{2}(x):=\sum_{i=1}^{n} \bar{\theta}_{i}^{*}\left\langle z_{i}, x\right\rangle+\bar{b}_{2} \\
& f_{3}(x):=\sum_{i=1}^{n} \bar{\kappa}_{i}^{*}\left\langle z_{i}, x\right\rangle+\bar{b}_{3}
\end{aligned}
$$


where $z_{1}, z_{2}, \ldots, z_{n}$ are the variables in the data set for regression, and $x=\left(x_{1}, x_{2}, x_{3}, x_{4}\right)^{T}$ indicates the independent variable with hot-pressing temperature $x_{1}$, hot-pressing pressure $x_{2}$, hot-pressing time $x_{3}$, and moisture content $x_{4}$.

The regression functions in (19) satisfy the following two properties.

(i) $f_{i}$ is continuously differentiable on $R^{n}, i=1,2,3$.

(ii) $f_{i}$ and $-f_{i}$ are not convex, but $-f_{i}$ is pseudoconvex on $R^{n}, i=1,2,3$.

And the regression functions in (20) satisfy the following two properties.

(i) $f_{i}$ is continuously differentiable on $R^{n}, i=1,2,3$.

(ii) $f_{i}$ and $-f_{i}$ are convex on $R^{n}, i=1,2,3$.

From the physical significance of MOR, MOS, and IBS, we suppose that the larger the numbers of MOR, MOS, and IBS, the better the quality of hot-pressing plate. In order to adopt the different demand on the indexes of the hot-pressing plate in different applications, we consider the following two cases in this part.

First, we focus on maximizing a single performance index of the hot-pressing plate when the other two performance indexes are within the certain areas. If we want to optimize the IBS, the corresponding optimization model for this case can be expressed by

$$
\begin{array}{ll}
\min & -f_{3}(x) \\
\text { s.t. } & 0 \leq x_{1} \leq v_{1}, \\
& 0 \leq x_{2} \leq v_{2}, \\
& 0 \leq x_{3} \leq v_{3}, \\
& 0 \leq x_{4} \leq v_{4}, \\
& -\iota_{1} \leq f_{1}(x)-y_{1}^{*} \leq \varepsilon_{1}, \\
& -\iota_{2} \leq f_{2}(x)-y_{2}^{*} \leq \varepsilon_{2},
\end{array}
$$

where $v_{1}, v_{2}, v_{3}, v_{4}>0$ indicate the upper bounds of hotpressing temperature, hot-pressing pressure, hot-pressing time, and moisture content, and $\left[y_{1}^{*}-\iota_{1}, y_{1}^{*}+\varepsilon_{1}\right],\left[y_{2}^{*}-\iota_{2}, y_{2}^{*}+\right.$ $\varepsilon_{2}$ ] are the feasible regions of MOR and MOS, respectively.

In order to let problem (21) be solved effectively, we let the objective function $f_{3}$ be with the Gaussian radial basis function, and the regression functions $f_{1}$ and $f_{2}$ in the constraints are with the linear polynomial kernel. Then, (21) is

$$
\min -\sum_{i=1}^{n} \kappa_{i}^{*} \exp \left(-\frac{\left\|z_{i}-x\right\|^{2}}{2 \sigma^{2}}\right)-b_{3}
$$

$$
\begin{array}{ll}
\text { s.t. } & 0 \leq x_{1} \leq v_{1}, \\
& 0 \leq x_{2} \leq v_{2}, \\
& \leq x_{3} \leq v_{3}, \\
& \leq x_{4} \leq \nu_{4}, \\
& -\iota_{1} \leq \sum_{i=1}^{n} \bar{\rho}_{i}^{*}\left\langle z_{i}, x\right\rangle+b_{1}-y_{1}^{*} \leq \varepsilon_{1}, \\
& -\iota_{2} \leq \sum_{i=1}^{n} \bar{\theta}_{i}^{*}\left\langle z_{i}, x\right\rangle+b_{2}-y_{2}^{*} \leq \varepsilon_{2},
\end{array}
$$

which is a pseudoconvex optimization problem with convex constraints.

Second, we would like to optimize the MOR, MOS, and IBS synthetically. For this demand, we consider the following optimization model:

$$
\begin{array}{ll}
\min & -\frac{\lambda_{1}}{f_{1}^{*}} f_{1}(x)-\frac{\lambda_{2}}{f_{2}^{*}} f_{2}(x)-\frac{\lambda_{3}}{f_{3}^{*}} f_{3}(x) \\
\text { s.t. } & 0 \leq x_{1} \leq v_{1}, \\
& 0 \leq x_{2} \leq v_{2}, \\
& 0 \leq x_{3} \leq v_{3}, \\
& 0 \leq x_{4} \leq v_{4},
\end{array}
$$

where $\lambda_{1}, \lambda_{2}, \lambda_{3}>0$ indicate the importance of MOR, MOS, and IBS, $v_{1}, v_{2}, v_{3}, v_{4}$ are with the same meaning as in (21), and $f_{1}^{*}, f_{2}^{*}$, and $f_{3}^{*}$ are the expected values of MOR, MOS, and IBS. In particular, if MOR, MOS, and IBS are with the same importance in the quality of the hot-pressing plate, we can let $\lambda_{1}=\lambda_{2}=\lambda_{3}=1 / 3$, and we can let $\lambda_{1}=1 / 2$, $\lambda_{2}=1 / 3$, and $\lambda_{3}=1 / 6$, if the importance of MOR, MOS, and IBS is strictly monotone decreasing. Similar to the kernel functions in problem (21), we let $f_{1}, f_{2}$, and $f_{3}$ in problem (23) be with the Gaussian radial basis function, which means that problem (23) is also a pseudoconvex optimization problem with convex constraints.

3.3. General Model. Based on analysis in Sections 3.1 and 3.2 , we consider the following minimization problem in this paper:

$$
\begin{array}{ll}
\text { minimize } & f(x):=\Theta(x)+\gamma \sum_{i=1}^{n}\left|x_{i}\right|+\sigma\left|e^{T} x\right| \\
\text { subject to } & x \in \Omega:=\{x: a \leq x \leq b\}, \\
& g_{i}(x) \leq 0, \quad i=1, \ldots, m,
\end{array}
$$


where $\gamma, \sigma \geq 0, a, b \in R^{n}$ with $a<b, \Theta: R^{n} \rightarrow R$ is continuously differentiable and pseudoconvex on $R^{n}$, and $g_{i}: R^{n} \rightarrow R(i=1,2, \ldots, m)$ is continuously differentiable and convex on $R^{n}$.

On the one hand, when $\Theta(x):=(1 / 2) x^{T} Q x-y^{T} x, a:=$ $-C e, b:=C e$, and $\gamma$ and $\sigma$ are defined as in (18), then problem (24) without $g_{i}$ reduces to problem (18). On the other hand, if we let

$$
\begin{aligned}
& \Theta(x):=-\sum_{i=1}^{n} \kappa_{i}^{*} \exp \left(-\frac{\left\|z_{i}-x\right\|^{2}}{2 \sigma^{2}}\right)-b_{3}, \\
& a:=0 \\
& b:=\left(\nu_{1}, \nu_{2}, v_{3}, v_{4}\right)^{T} \\
& g_{1}(x):=-\sum_{i=1}^{n} \bar{\rho}_{i}^{*}\left\langle z_{i}, x\right\rangle-b_{1}+y_{1}^{*}-\iota_{1}, \\
& g_{2}(x):=\sum_{i=1}^{n} \bar{\rho}_{i}^{*}\left\langle z_{i}, x\right\rangle+b_{1}-y_{1}^{*}-\varepsilon_{1}, \\
& g_{3}(x):=-\sum_{i=1}^{n} \bar{\theta}_{i}^{*}\left\langle z_{i}, x\right\rangle-b_{2}+y_{2}^{*}-\iota_{2}, \\
& g_{4}(x):=\sum_{i=1}^{n} \bar{\theta}_{i}^{*}\left\langle z_{i}, x\right\rangle+b_{2}-y_{2}^{*}-\varepsilon_{2},
\end{aligned}
$$

then problem (24) reduces to problem (22). Similar reformulation can be done for problem (23) by (24).

Therefore, problem (24) considered in this paper includes the optimization models for building up the relationships and optimizing the relative parameters in MDF continuous hotpressing process.

In what follows, we denote $\mathscr{F}$ as the feasible region of (24); that is,

$$
\mathscr{F}:=\left\{x \in \Omega: g_{i}(x) \leq 0, i=1,2, \ldots, m\right\},
$$

and $\mathscr{M}$ is the optimal solution set of (24).

\section{Main Results}

4.1. Proposed Neural Network. In this subsection, we propose a one-layer recurrent neural network for solving problem (24), where we combine the penalty function and projection methods to solve the constraints and use the smoothing techniques to overcome the nonsmoothness of the objective function and penalty function.

Define the penalty function

$$
p(x)=\sum_{i=1}^{m} \max \left\{0, g_{i}(x)\right\} .
$$

Then $\{x \in \Omega: p(x) \leq 0\}=\mathscr{F}$.

From the smoothing functions in (25) for the plus function, we define the smoothing function of $p$ as

$$
\widetilde{p}(x, \mu)=\sum_{i=1}^{m} \phi\left(g_{i}(x), \mu\right),
$$

where

$$
\phi(s, \mu)= \begin{cases}\max \{0, s\} & \text { if }|s|>\mu \\ \frac{(s+\mu)^{2}}{4 \mu} & \text { if }|s| \leq \mu .\end{cases}
$$

Form the results in [23], $\phi(s, \mu)$ owns the following properties.

Lemma 3 (see [23]). (i) For any $s \in R, \phi(s, \cdot)$ is continuously differentiable, and $\phi(\cdot, \mu)$ is also continuously differentiable for any fixed $\mu>0$

(ii) $0 \leq \nabla_{\mu} \phi(s, \mu) \leq 1, \forall s \in R, \forall \mu \in(0,+\infty)$;

(iii) $0 \leq \phi(s, \mu)-|s| \leq \mu / 4, \forall s \in R, \forall \mu \in(0,+\infty)$;

(iv) $\phi(\cdot, \mu)$ is convex for any fixed $\mu>0$, and $\left\{\lim _{s \rightarrow t, \mu \downarrow 0} \nabla_{s} \phi(s, \mu)\right\} \subseteq \partial \max \{0, t\}$

Then, $\widetilde{p}$ has the following properties.

Lemma 4. $\widetilde{p}$ is a smoothing function $p$ and satisfies the following:

(i) $\widetilde{p}(\cdot, \mu)$ is convex for any fixed $\mu>0$;

(ii) $\left\{\lim _{z \rightarrow x, \mu \downarrow 0} \nabla_{z} \tilde{p}(z, \mu)\right\} \subseteq \partial p(x)$;

(iii) $0 \leq \nabla_{\mu} \widetilde{p}(x, \mu) \leq m, \forall x \in R^{n}, \mu \in(0,+\infty)$.

Next, by the smoothing function for the absolute value function $|\cdot|$

$$
\theta(s, \mu)= \begin{cases}|s| & \text { if }|s| \geq \mu \\ \frac{s^{2}}{2 \mu}+\frac{\mu}{2} & \text { if }|s|<\mu\end{cases}
$$

we define

$$
\widetilde{f}(x, \mu):=\Theta(x)+\gamma \sum_{i=1}^{n} \theta\left(x_{i}, \mu\right)+\sigma \theta\left(e^{T} x, \mu\right) .
$$

Since $\theta(s, \mu)=\phi(s, \mu)+\phi(-s, \mu), \theta(s, \mu)$ owns all properties in Lemma 8 and the following results hold.

Lemma 5. $\tilde{f}$ is a smoothing function $f$ in (24) with the following properties:

(i) $\widetilde{f}(\cdot, \mu)$ is pseudoconvex for any fixed $\mu>0$;

(ii) $\left\{\lim _{z \rightarrow x, \mu \downarrow 0} \nabla_{x} \tilde{f}(x, \mu)\right\} \subseteq \partial f(x)$;

(iii) $0 \leq \nabla_{\mu} \tilde{f}(x, \mu) \leq \gamma n+\sigma, \forall x \in R^{n}, \mu \in(0,+\infty)$.

From the projected gradient method and the viscosity regularization method, we introduce the following neural network to solve (24):

$$
\begin{aligned}
& \dot{x}(t) \in-x(t)+P_{\Omega}\left[x(t)-\nabla_{x} \tilde{p}(x(t), \mu(t))\right. \\
& \left.-\varepsilon(t) \nabla_{x} \tilde{f}(x(t), \mu(t))\right],
\end{aligned}
$$

where $\mu(t)=e^{-t}$ and $\varepsilon(t)=1 /(t+1)$ with $\varepsilon_{0}>0$. 


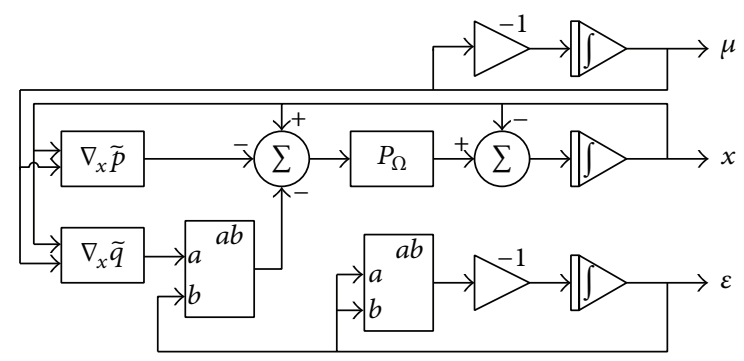

FIGURE 1: Simple block structure of proposed network (37).

By the definitions for $\widetilde{f},(34)$ can be expressed as

$$
\begin{aligned}
& \dot{x}(t) \in-x(t)+P_{\Omega}[x(t) \\
& -\sum_{i=1}^{r} \nabla_{s} \phi(s, \mu(t))_{s=g_{i}(x(t))} \nabla g_{i}(x(t))-\varepsilon(t) \\
& \quad\left(\nabla \Theta(x(t))+\gamma \sum_{i=1}^{n} \nabla_{s} \theta(s, \mu)_{s=x_{i}(t)} e_{i}\right. \\
& \left.\left.+\sigma \nabla_{s} \theta(s, \mu)_{s=e^{T} x(t)} e\right)\right],
\end{aligned}
$$

where

$$
\begin{aligned}
& \nabla_{s} \phi(s, \mu)= \begin{cases}1 & \text { if } s>\mu \\
\frac{s+\mu}{2 \mu} & \text { if }|s| \leq \mu \\
0 & \text { if } s<-\mu,\end{cases} \\
& \nabla_{s} \theta(s, \mu)= \begin{cases}1 & \text { if }|s| \geq \mu \\
\frac{s}{\mu} & \text { if }|s|<\mu .\end{cases}
\end{aligned}
$$

To implement (34) by circuits, we can use the reformulated form of (34) as follows:

$$
\begin{aligned}
\dot{x}(t) & \in-x(t)+P_{\Omega}\left[x(t)-\nabla_{x} \widetilde{p}(x(t), \mu(t))\right. \\
& \left.-\varepsilon(t) \nabla_{x} \widetilde{f}(x(t), \mu(t))\right], \\
\dot{\varepsilon}(t) & =-\varepsilon^{2}(t), \\
\dot{\mu}(t) & =-\mu(t) .
\end{aligned}
$$

Equation (34) can be seen as a network with three input and three output variables that are $x(t), \mu(t)$, and $\varepsilon(t)$. A simple block structure of the proposed network (37) implemented by circuits is presented in Figure 1.

4.2. Theoretical Analysis. In this subsection, we study some necessary dynamical and optimality properties of proposed network (34) for solving (24).

The global existence of the solutions of (34) is a necessary condition for its usability in optimization. With an initial point $x_{0} \in \Omega$, the solution of (34) is global existent. Moreover, the uniqueness of the solution of (34) with $x_{0} \in \Omega$ is proved under some conditions. The proposed network (34) can be implemented in circuits and mathematical software. Then, the feasibility and optimality of network (34) for optimization problem (24) are proved theoretically.

We call $x:[0, T)$ with $\mathrm{T}>0$ a solution of (34) if $x$ is absolutely continuous on $[0, T)$ and satisfies (34) everywhere.

Proposition 6. For any initial point $x_{0} \in \Omega$, there is a global solution of (34) defined on $[0,+\infty)$ and it satisfies

$$
x(t) \in \Omega, \quad \forall t \in[0,+\infty) .
$$

Proof. Since the right hand function in network (34) is continuous about $x$ and $t$, there are $T>0$ and an absolute continuous function $x:[0, T) \rightarrow R^{n}$ such that $x(t)$ satisfies (34) for all $t \in[0, T)$.

Denote $\theta(t)=P_{\Omega}\left[x(t)-\nabla_{x} \tilde{p}(x(t), \mu(t))-\varepsilon(t) \nabla_{x} \tilde{f}(x(t)\right.$, $\mu(t))$ ]. Then, (34) can be rewritten as

$$
\dot{x}(t)+x(t)=\theta(t), \quad t \in[0, T) .
$$

A simple integration procedure of the above equation gives

$$
x(t)=e^{-t} x_{0}+e^{-t} \int_{0}^{t} \theta(s) e^{s} d s
$$

which can be rewritten as

$$
x(t)=e^{-t} x_{0}+\left(1-e^{-t}\right) \int_{0}^{t} \theta(s) \frac{e^{s}}{e^{t}-1} d s .
$$

By $\int_{0}^{t} e^{s} /\left(e^{t}-1\right) d s=1, \theta(s) \in \Omega, \forall 0 \leq s \leq t$ and $x_{0} \in \Omega$, we obtain that $x(t) \in \Omega, \forall t \in[0, T)$.

By the boundedness of $\Omega$ and the extension theory, this solution of (34) can be extended. Thus, the solution of (34) with initial point $x_{0} \in \Omega$ is globally existent. Similarly, we can obtain that

$$
x(t) \in \Omega, \quad \forall t \in[0,+\infty) .
$$

Some Lipschitz condition is often used to guarantee the uniqueness of the solution of a neural network. In what follows, we give a sufficient condition to ensure the uniqueness of the solution of (34) with initial point $x_{0} \in \Omega$.

Proposition 7. For any initial point $x_{0} \in \Omega$, if $\nabla_{x} \widetilde{f}(\cdot, \mu)$ and $\nabla_{x} \widetilde{p}(\cdot, \mu)$ are locally Lipschitz continuous for any fixed $\mu \epsilon$ $(0,1]$, then the solution of neural network (34) is unique.

Proof. By Proposition 6, the solutions of (34) with initial point $x_{0} \in \Omega$ exist globally and satisfy $x(t) \in \Omega, \forall t \in[0,+\infty)$. Suppose that there exist two solutions $x:[0,+\infty) \rightarrow R^{n}$ and $y:[0,+\infty) \rightarrow R^{n}$ of (34) with initial point $x_{0} \in \Omega$, and suppose there exists $\widehat{t}$ such that $\widehat{t}=\inf _{t \geq 0, x(t) \neq y(t)} t$. By the boundedness of $\Omega$, there is $R>0$ such that $\|x(t)\| \leq R$ and $\|y(t)\| \leq R, \forall t \in[0,+\infty)$. 
Then, there is $L>0$ such that

$$
\begin{array}{r}
\left|\nabla_{x} \tilde{f}(x(t), \mu(t))-\nabla_{x} \tilde{f}(y(t), \mu(t))\right| \\
\leq L\|x(t)-y(t)\|, \quad \forall t \in[\widehat{t}, \widehat{t}+1], \\
\left|\nabla_{x} \widetilde{p}(x(t), \mu(t))-\nabla_{x} \widetilde{p}(y(t), \mu(t))\right| \\
\leq L\|x(t)-y(t)\|, \quad \forall t \in[\widehat{t}, \hat{t}+1] .
\end{array}
$$

Differentiating $(1 / 2)\|x(t)-y(t)\|^{2}$ along the two solutions of (34), by the Lipschitz continuity of $P_{\Omega}$ and (43), we have

$$
\begin{aligned}
\frac{d}{d t} \frac{1}{2}\|x(t)-y(t)\|^{2} & =\langle x(t)-y(t), \dot{x}(t)-\dot{y}(t)\rangle \\
& \leq(L+\varepsilon(t) L)\|x(t)-y(t)\|^{2} .
\end{aligned}
$$

Applying Gronwall's inequality into the integration of the above inequality, it gives $x(t)=y(t), \forall t \in[\widehat{t}, \widehat{t}+1]$, which leads to a contradiction. Therefore, the solution of (34) with initial point $x_{0} \in \Omega$ is unique.

Lyapunov method is employed to analyze the performance of (34). Here, we introduce the following two Lyapunov energy functions:

$$
\begin{aligned}
E(x, t)= & \widetilde{p}(x, \mu(t))+\varepsilon(t)\left(\tilde{f}(x, \mu(t))-\inf _{\Omega} f\right), \\
G(x, t)= & \frac{1}{2} \operatorname{dist}^{2}(x, \mathscr{M})+\widetilde{p}(x, \mu(t)) \\
& +\varepsilon(t)\left(\tilde{f}(x, \mu(t))-\inf _{\Omega} f\right) .
\end{aligned}
$$

The above two Lyapunov functions satisfy the following estimations along the solutions of (34).

Lemma 8. (i) The derivative of $E(x, t)$ along the solution of (34) can be calculated by

$$
\frac{d}{d t} E(x(t), t) \leq-\|\dot{x}(t)\|^{2} .
$$

(ii) The derivative of $G(x, t)$ along the solution of (34) can be calculated by

$$
\begin{aligned}
& \frac{d}{d t} G(x(t), t) \leq-\|\dot{x}(t)\|^{2}-\left\langle\nabla_{x} \widetilde{p}(x(t), \mu(t))\right. \\
& \left.\quad+\varepsilon(t) \nabla_{x} \tilde{f}(x(t), \mu(t)), x(t)-P_{\mathscr{M}}(x(t))\right\rangle .
\end{aligned}
$$

Proof. (i) Differentiating $E(x(t), t)$ along the solutions of (34), we have

$$
\begin{aligned}
& \frac{d}{d t} E(x(t), t)=\left\langle\nabla_{x} \widetilde{p}(x(t), \mu(t))\right. \\
& \left.\quad+\varepsilon(t) \nabla_{x} \tilde{f}(x(t), \mu(t)), \dot{x}(t)\right\rangle+\dot{\varepsilon}(t) \\
& \quad \cdot\left(\tilde{f}(x(t), \mu(t))-\inf _{\Omega} f\right)+\left(\nabla_{\mu} \tilde{p}(x(t), \mu(t))\right. \\
& \left.\quad+\varepsilon(t) \nabla_{\mu} \tilde{f}(x(t), \mu(t))\right) \dot{\mu}(t) .
\end{aligned}
$$

Equation (34) can be rewritten as

$$
\begin{aligned}
\dot{x}(t) & +x(t)=P_{\Omega}\left[x(t)-\nabla_{x} \tilde{p}(x(t), \mu(t))\right. \\
& \left.-\varepsilon(t) \nabla_{x} \tilde{f}(x(t), \mu(t))\right] .
\end{aligned}
$$

Letting $v=x(t)-\nabla_{x} \widetilde{p}(x(t), \mu(t))-\varepsilon(t) \nabla_{x} \widetilde{f}(x(t), \mu(t))$ and $u=x(t)$, using (49) and (10), we have

$$
\begin{aligned}
& \left\langle x(t)-\nabla_{x} \tilde{p}(x(t), \mu(t))-\varepsilon(t) \nabla_{x} \tilde{f}(x(t), \mu(t))\right. \\
& -\dot{x}(t)-x(t), \dot{x}(t)+x(t)-x(t)\rangle \geq 0,
\end{aligned}
$$

which follows the fact that

$$
\begin{aligned}
& \left\langle\nabla_{x} \tilde{p}(x(t), \mu(t))+\varepsilon(t) \nabla_{x} \tilde{f}(x(t), \mu(t)), \dot{x}(t)\right\rangle \\
& \quad \leq-\|\dot{x}(t)\|^{2}, \quad \forall t \geq 0 .
\end{aligned}
$$

Combining (48) and (51), we get that

$$
\begin{aligned}
& \frac{d}{d t} E(x(t), t) \leq-\|\dot{x}(t)\|^{2}+\dot{\varepsilon}(t) \\
& \quad \cdot\left(\tilde{f}(x(t), \mu(t))-\inf _{\Omega} f\right) \\
& \quad+\left(\nabla_{\mu} \tilde{p}(x(t), \mu(t))+\varepsilon(t) \nabla_{\mu} \tilde{f}(x(t), \mu(t))\right) \dot{\mu}(t) .
\end{aligned}
$$

From Lemmas 4 and $5, \dot{\mu}(t)=-e^{-t}$ and $\dot{\varepsilon}(t)<0$, we obtain the estimation in (i). we have

(ii) Differentiating $G(x(t), t)$ along the solutions of (34),

$$
\begin{aligned}
& \frac{d}{d t} G(x(t), t)=\left\langle x(t)-P_{M}(x(t)), \dot{x}(t)\right\rangle \\
& \quad+\left\langle\nabla_{x} \tilde{p}(x(t), \mu(t))\right. \\
& \left.\quad+\varepsilon(t) \nabla_{x} \tilde{f}(x(t), \mu(t)), \dot{x}(t)\right\rangle+\dot{\varepsilon}(t) \\
& \quad \cdot\left(\tilde{f}(x(t), \mu(t))-\inf _{\Omega} f\right)+\left(\nabla_{\mu} \tilde{p}(x(t), \mu(t))\right. \\
& \left.\quad+\varepsilon(t) \nabla_{\mu} \tilde{f}(x(t), \mu(t))\right) \dot{\mu}(t) .
\end{aligned}
$$

Letting $v=x(t)-\nabla_{x} \widetilde{p}(x(t), \mu(t))-\varepsilon(t) \nabla_{x} \tilde{f}(x(t), \mu(t))$ and $u=P_{\mathscr{M}}(x(t))$, using (10) and (49), we have

$$
\begin{gathered}
\left\langle\nabla_{x} \tilde{p}(x(t), \mu(t))+\varepsilon(t) \nabla_{x} \tilde{f}(x(t), \mu(t))\right. \\
\left.\quad+\dot{x}(t), \dot{x}(t)+x(t)-P_{\mathscr{M}}(x(t))\right\rangle \leq 0,
\end{gathered}
$$

which can be rewritten as

$$
\begin{aligned}
& \left\langle\nabla_{x} \tilde{p}(x(t), \mu(t))+\varepsilon(t) \nabla_{x} \tilde{f}(x(t), \mu(t)), \dot{x}(t)\right\rangle \\
& \quad+\left\langle\dot{x}(t), x(t)-P_{\mathscr{M}}(x(t))\right\rangle \\
& \quad \leq-\left\langle\nabla_{x} \tilde{p}(x(t), \mu(t))\right. \\
& \left.\quad+\varepsilon(t) \nabla_{x} \tilde{f}(x(t), \mu(t)), x(t)-P_{\mathscr{M}}(x(t))\right\rangle \\
& \quad-\|\dot{x}(t)\|^{2} .
\end{aligned}
$$


Combining (53) and (55), we get

$$
\begin{aligned}
& \frac{d}{d t} G(x(t), t) \leq-\|\dot{x}(t)\|^{2}+\dot{\varepsilon}(t)\left(f(x(t))-\inf _{\Omega} f\right) \\
& \quad+\left(\nabla_{\mu} \widetilde{p}(x(t), \mu(t))+\varepsilon(t) \nabla_{\mu} \tilde{f}(x(t), \mu(t))\right) \dot{\mu}(t) \\
& \quad-\left\langle\nabla_{x} \widetilde{p}(x(t), \mu(t))\right. \\
& \left.\quad+\varepsilon(t) \nabla_{x} \tilde{f}(x(t), \mu(t)), x(t)-P_{\mathscr{M}}(x(t))\right\rangle .
\end{aligned}
$$
(ii).

Similar to the analysis in (i), we obtain the estimation in

Next, we prove the efficiency of proposed network (34) for solving optimization problem (24), where the convergence feasibility of the proposed network is a basic property.

Theorem 9. For initial point $x_{0} \in \Omega$, any solution $x:[0,+\infty)$ $\rightarrow R^{n}$ of (34) satisfies

$$
\lim _{t \rightarrow+\infty} \operatorname{dist}(x(t), \mathscr{F})=0 .
$$

Proof. Denote $x:[0,+\infty) \rightarrow R^{n}$ as a global solution of (34) with initial point $x_{0} \in \Omega$.

From $x(t) \in \Omega, \forall t \in[0,+\infty)$ and Lemma $8, E(x(t), t)$ is nonincreasing along the solution of (34). Using $E(x(t), t) \geq 0$, $\forall t \in[0,+\infty)$, we confirm that

$$
\lim _{t \rightarrow+\infty} E(x(t), t) \text { exists. }
$$
have

From $x(t) \in \Omega, \forall t \in[0,+\infty)$ and $\lim _{t \rightarrow+\infty} \varepsilon(t)=0$, we

$$
\lim _{t \rightarrow+\infty} \varepsilon(t)\left(\tilde{f}(x(t), \mu(t))-\inf _{\Omega} f\right)=0
$$

which implies

$$
\lim _{t \rightarrow+\infty} E(x(t), t)=\lim _{t \rightarrow+\infty} \widetilde{p}(x(t), \mu(t)) .
$$

In what follows, we will prove that $\lim _{t \rightarrow+\infty} \widetilde{p}(x(t), \mu(t))=$ 0 . Arguing by contradiction, we assume that

$$
\lim _{t \rightarrow+\infty} \widetilde{p}(x(t), \mu(t))=c \neq 0 .
$$

By $\widetilde{p}(x(t), \mu(t)) \geq p(x(t)) \geq 0, \forall t \in[0,+\infty)$, we have $c>0$, which follows the fact that there is $T_{1} \geq 0$ such that

$$
\widetilde{p}(x(t), \mu(t)) \geq \frac{c}{2}, \quad \forall t \in\left[T_{1},+\infty\right) .
$$

From Lemma 4, we obtain

$$
\left|\widetilde{p}\left(P_{\mathscr{M}}(x(t)), \mu(t)\right)-p\left(P_{\mathscr{M}}(x(t))\right)\right| \leq m \mu(t),
$$

which implies that there exists $T_{2}>T_{1}$ such that

$$
\widetilde{p}\left(P_{\mu}(x(t)), \mu(t)\right) \leq \frac{c}{8} .
$$

Since $\Omega$ is bounded and $x(t) \in \Omega, \forall t \in[0,+\infty)$, by Lemma 5 , there is $R>0$ such that

$$
\begin{aligned}
&\left|\left\langle\nabla_{x} \tilde{f}(x(t), \mu(t)), x(t)-P_{\mathscr{M}}(x(t))\right\rangle\right| \leq R, \\
& \forall t \in[0,+\infty) .
\end{aligned}
$$

Then,

$$
\lim _{t \rightarrow+\infty} \varepsilon(t)\left\langle\nabla_{x} \tilde{f}(x(t), \mu(t)), x(t)-P_{\mathscr{M}}(x(t))\right\rangle=0,
$$

which implies that there is $T_{3} \geq T_{2}$ such that

$$
\begin{array}{r}
\left|\varepsilon(t)\left\langle\nabla_{x} \tilde{f}(x(t), \mu(t)), P_{\mathscr{M}}(x(t))-x(t)\right\rangle\right| \leq \frac{c}{8}, \\
\forall t \in\left[T_{2},+\infty\right) .
\end{array}
$$

From Lemma 8 and the convexity of $\widetilde{p}(\cdot, \mu)$ for any fixed $\mu>0$, we obtain that

$$
\begin{aligned}
& \frac{d}{d t} G(x(t), t) \leq-\left\langle\nabla_{x} \widetilde{p}(x(t), \mu(t))\right. \\
& \left.\quad+\varepsilon(t) \nabla_{x} \tilde{f}(x(t), \mu(t)), x(t)-P_{\mathscr{M}}(x(t))\right\rangle \\
& \quad \leq-\widetilde{p}(x(t), \mu(t))+\widetilde{p}\left(P_{\mathscr{M}}(x(t)), \mu(t)\right)-\varepsilon(t) \\
& \cdot\left\langle\nabla_{x} \tilde{f}(x(t), \mu(t)), x(t)-P_{\mathscr{M}}(x(t))\right\rangle .
\end{aligned}
$$

By (62), (67), and (68), we obtain

$$
\frac{d}{d t} G(x(t), t) \leq-\frac{c}{4}, \quad \forall t \in\left[T_{3},+\infty\right)
$$

Integrating the above inequality from $T_{3}$ to $t\left(>T_{3}\right)$, we have

$$
\begin{aligned}
& G(x(t), t) \leq G\left(x\left(T_{3}\right), T_{3}\right)-\frac{c}{4}\left(t-T_{3}\right) \\
& \forall t \in\left[T_{3},+\infty\right) .
\end{aligned}
$$

Thus,

$$
\lim _{t \rightarrow+\infty} G(x(t), t)=-\infty,
$$

which leads to a contradiction with $G(x, t) \geq 0$ for all $x \in R^{n}$ and $t \in[0,+\infty)$. Therefore,

$$
\lim _{t \rightarrow+\infty} p(x(t))=\lim _{t \rightarrow+\infty} \tilde{p}(x(t), \mu(t))=0,
$$

which guarantees that

$$
\lim _{t \rightarrow+\infty} \operatorname{dist}(x(t), \mathscr{F})=0 .
$$

The following theorem indicates that any accumulation point of the solutions of (34) is just an optimal solution of (34). 
Theorem 10. For initial point $x_{0} \in \Omega$, any solution $x$ of (34) is convergent to the optimal solution set $\mathscr{M}$; that is,

$$
\lim _{t \rightarrow+\infty} \operatorname{dist}(x(t), \mathscr{M})=0 .
$$

Proof. From (63), (68), and $\widetilde{p}(x(t), \mu(t)) \geq 0, \forall t \in[0,+\infty)$, we have

$$
\begin{aligned}
& \frac{d}{d t} G(x(t), t) \\
& \leq-\varepsilon(t)\left\langle\nabla_{x} \widetilde{f}(x(t), \mu(t)), x(t)-P_{\mathscr{M}}(x(t))\right\rangle \\
& \quad+m \mu(t),
\end{aligned}
$$

by $\dot{\mu}(t)=-\mu(t)$, which can be rewritten as

$$
\begin{aligned}
& \frac{d}{d t}[G(x(t), t)+m \mu(t)] \\
& \quad \leq-\varepsilon(t)\left\langle\nabla_{x} \tilde{f}(x(t), \mu(t)), x(t)-P_{\mathscr{M}}(x(t))\right\rangle .
\end{aligned}
$$

Denote

$$
\begin{aligned}
I= & \{t \\
& \in[0,+\infty):\left\langle\nabla_{x} \widetilde{f}(x(t), \mu(t)), x(t)-P_{\mathscr{M}}(x(t))\right\rangle \\
& \leq 0\} ; \\
J= & \{t \\
& \in[0,+\infty):\left\langle\nabla_{x} \widetilde{f}(x(t), \mu(t)), x(t)-P_{\mathscr{M}}(x(t))\right\rangle \\
& >0\} .
\end{aligned}
$$

Owning to the continuity of $\left\langle\nabla_{x} \tilde{f}(x(t), \mu(t)), x(t)-P_{\mathscr{M}}(x(t))\right\rangle$ on $[0,+\infty), I$ and $J$ are closed and open in $[0,+\infty)$, respectively.

Case 1. In this case, we assume that there exists $T \geq 0$ such that $t \in I, \forall t \in[T,+\infty)$.

From the definition on $I$ and the pseudoconvexity of $\tilde{f}(\cdot, \mu)$ on $R^{n}$, we have

$$
\tilde{f}(x(t), \mu(t)) \leq \tilde{f}\left(P_{\mathscr{M}}(x(t)), \mu(t)\right),
$$

which implies

$$
\forall t \in[T,+\infty),
$$

$$
\limsup _{t \rightarrow+\infty} f(x(t)) \leq \min _{x \in \mathscr{F}} f(x)
$$

By Theorem 9, we confirm that

$$
\lim _{t \rightarrow+\infty} \operatorname{dist}(x(t), \mathscr{M})=0 .
$$

Case 2. In this case, we assume that there exists $T \geq 0$ such that $t \in J, \forall t \in[T,+\infty)$, which means that

$$
\frac{d}{d t}[G(x(t), t)+m \mu(t)]<0, \quad \forall t \in[T,+\infty) .
$$

Then,

$$
\lim _{t \rightarrow+\infty}[G(x(t), t)+m \mu(t)] \text { exists. }
$$

Since $\lim _{t \rightarrow+\infty} E(x(t), t)$ exists and $\lim _{t \rightarrow+\infty} \mu(t)=0$, we obtain that

$$
\lim _{t \rightarrow+\infty} \operatorname{dist}(x(t), \mathscr{M}) \text { exists. }
$$

Suppose

$$
\begin{aligned}
& \liminf _{t \rightarrow+\infty}\left\langle\nabla_{x} \tilde{f}(x(t), \mu(t)), x(t)-P_{\mathscr{M}}(x(t))\right\rangle=d \\
& \quad>0 .
\end{aligned}
$$

Then, there is $T_{1} \geq$ such that

$$
\begin{aligned}
\left\langle\nabla_{x} \tilde{f}(x(t), \mu(t)), x(t)-P_{\mathscr{M}}(x(t))\right\rangle & \geq \frac{d}{2}, \\
\forall t & \in\left[T_{1},+\infty\right) .
\end{aligned}
$$

Then, from (76), we have

$$
\begin{aligned}
\frac{d}{d t}[G(x(t), t)+m \mu(t)] \leq-\frac{d}{2} \varepsilon(t), & \\
& \forall t \in\left[T_{1},+\infty\right) .
\end{aligned}
$$

Integrating the above inequality from $T_{1}$ to $t\left(>T_{1}\right)$, we have

$$
\begin{aligned}
G(x(t), t)+m \mu(t) \leq & G\left(x\left(T_{1}\right), T_{1}\right)+m \mu\left(T_{1}\right) \\
& +\int_{T_{1}}^{t}-\frac{d}{2} \varepsilon(s) d s .
\end{aligned}
$$

Let $t \rightarrow+\infty$ in the above inequality; then we have

$$
\lim _{t \rightarrow+\infty} G(x(t), t)=-\infty,
$$

which leads to a contraction with the boundedness from below $G(x(t), t)$ on $[0,+\infty)$. Thus,

$$
\liminf _{t \rightarrow+\infty}\left\langle\nabla_{x} \tilde{f}(x(t), \mu(t)), x(t)-P_{\mathscr{M}}(x(t))\right\rangle=0,
$$

which follows the fact that there is an increasing sequence $\left\{t_{n}\right\}$ such that $t_{n} \rightarrow+\infty$ and

$$
\begin{aligned}
& \lim _{n \rightarrow+\infty}\left\langle\nabla_{x} \tilde{f}\left(x\left(t_{n}\right), \mu\left(t_{n}\right)\right), x\left(t_{n}\right)-P_{\mathscr{M}}\left(x\left(t_{n}\right)\right)\right\rangle \\
& \quad=0 .
\end{aligned}
$$

By $\left\{x\left(t_{n}\right)\right\} \subseteq \Omega$ and $\Omega$ which is bounded, there are $x^{* *} \epsilon$ $\Omega$ and a subsequence of $\left\{x\left(t_{n}\right)\right\}$ (denoted as $\left\{x\left(t_{n_{k}}\right)\right\}$ ) such that $\lim _{k \rightarrow+\infty} t_{n_{k}}=+\infty$ and $\lim _{k \rightarrow+\infty} x\left(t_{n_{k}}\right)=x^{* *}$. By Lemma 5 , there is $\xi\left(x^{* *}\right) \in \partial f\left(x^{* *}\right)$ such that

$$
\left\langle\xi\left(x^{* *}\right), x^{* *}-P_{\mathscr{M}}\left(x^{* *}\right)\right\rangle=0 .
$$

From the pseudoconvexity of $f$ and the above inequality, we have

$$
f\left(x^{* *}\right) \leq f\left(P_{\mathscr{M}}\left(x^{* *}\right)\right) .
$$


TABLE 1: Experimental data set in MDF hot-pressing.

\begin{tabular}{lccccrr}
\hline Regression parameter & $\gamma$ & $\sigma$ & $C$ & MSE & DF & SE \\
\hline MOR & 0.001 & 5 & 30 & 0.0307 & 0.9543 & -0.8299 \\
MOE & 0.001 & 6 & 40 & 0.0642 & 0.9063 & -1.7060 \\
IBS & 0.001 & 5 & 40 & 0.0451 & 0.9256 & -0.6388 \\
\hline
\end{tabular}

By Theorem 9, we have $x^{* *} \in \mathscr{F}$. Therefore, $x^{* *} \in \mathscr{M}$, which implies that

$$
\lim _{k \rightarrow+\infty} \operatorname{dist}\left(x\left(t_{n_{k}}\right), \mathscr{M}\right)=0 .
$$

Combining the above results with (83), we conclude that $\lim _{t \rightarrow+\infty} \operatorname{dist}(x(t), \mathscr{M})=0$.

Case 3. In this case, we assume that both $I$ and $J$ are unbounded.

For $t \in I$, similar to the analysis in Case 1 , we have

$$
\lim _{t \rightarrow+\infty, t \in I} \operatorname{dist}(x(t), \mathscr{M})=0 .
$$

For $t \in J$, define $\tau(t)=\sup _{s \leq t, s \in I} s$. Then $\tau(t) \in I$ and $(\tau(t), t] \subseteq J$. By the unboundedness of $I$ and $J$, we have

$$
\lim _{t \in J, t \rightarrow+\infty} \tau(t)=+\infty .
$$
have

From (81) and the continuity of $G(x(t), t)$ on $[0,+\infty)$, we

$$
\begin{aligned}
G(x(t), t)+m \mu(t) \leq & G(x(\tau(t)), \tau(t)) \\
& +m \mu(\tau(t)), \quad \forall t \in J .
\end{aligned}
$$

By $\tau(t) \in I,(94)$, and (95), we have

$$
\begin{aligned}
& \limsup _{t \rightarrow+\infty, t \in J}[G(x(t), t)+m \mu(t)] \\
& \quad \leq \lim _{t \rightarrow+\infty, t \in J}[G(x(\tau(t)), \tau(t))+m \mu(\tau(t))]=0,
\end{aligned}
$$

which gives

$$
\lim _{t \rightarrow+\infty, t \in J} G(x(t), t)=0 .
$$

From the definition of $G(x, t)$ and the above result, we have

$$
\lim _{t \rightarrow+\infty, t \in J} \operatorname{dist}(x(t), \mathscr{M})=0 .
$$

Therefore, from (94) and (99), we obtain

$$
\lim _{t \rightarrow+\infty} \operatorname{dist}(x(t), \mathscr{M})=0 .
$$

\section{Numerical Experiments}

In this section, we test the proposed neural network (34) for solving problem (24), which is brought forward from the MDF continuous hot-pressing process. Based on the existing data set, we use the established theories and proposed neural network (34) to build the relationships between the main qualities of the hot-pressing plate and some relative technology parameters from optimization problem (18). Then, based on optimization problem (22), we will use proposed network (34) to solve the optimal values of the technology parameters in hot-pressing system for optimizing the qualities of the hot-pressing plate. All these numerical experiments validate the good performance of the proposed network in this paper.

The numerical testing was carried out on a Lenovo PC (3.00 GHz, 2.00 GB of RAM) with the use of Matlab 7.4. And we use ode23 to realize the neural network (34) in Matlab.

5.1. Construction Relation Models in MDF Continuous HotPressing Process. In this part, by considered optimization problem (18) and network (34), we build the relation model which takes the hot-pressing temperature (TE), hot-pressing pressure (PR), hot-pressing time (TI), and moisture content (MC) of slab as the argument variables and the MOR, MOE, and IBS indexes of MDF as the dependent variables. The numerical results show the good fitting of the built models for the data set, where the data set is given in Table 4 .

In order to use the data in Table 4, we first normalize them into $[0,1]$. And we use the mean square error (MSE), degree of fitting (DF), and sufficient evaluation (SE) to evaluate the numerical results, where

$$
\begin{aligned}
\text { MSE: MSE } & =\sqrt{\frac{\sum_{i=1}^{n}\left(x_{i}-x_{i}^{*}\right)^{2}}{n}}, \\
\text { DF: } \mathrm{DF} & =1-\sqrt{\left(\frac{\sum_{i=1}^{n}\left(x_{i}-x_{i}^{*}\right)^{2}}{\sum_{i=1}^{n} x_{i}^{2}}\right)}, \\
\text { SE: SE } & =\frac{1}{2} x^{T} Q x-y^{T} x,
\end{aligned}
$$

where $x$ and $x^{*}$ indicate the actual value and predicted value and $Q$ and $y$ are defined as in (18). The smaller the MSE and the SE and the closer the DF to 1, the better the regression result. Moreover, the SE function is the objective function in (15).

Based on the Gaussian radial basis function kernel, the values of the initial parameters in problem (18) are given in Table 1 . With a random initial point $x_{0} \in \Omega$, the numerical results with respect to the obtained solution are also listed in Table 1. Figures 2-4 illustrate the fitting effect of the MOR, MOE, and IBS values by using the proposed network (34) for solving problem (18). And the parameters of the regression functions in (19) are shown in Table 5. 
TABLE 2: Criterion of physical and mechanical performance indexes.

\begin{tabular}{lcccccrrr}
\hline Performance index & Unit & \multicolumn{5}{c}{ Thickness range } \\
& & $4 \sim 6$ & $6 \sim 9$ & $9 \sim 12$ & $12 \sim 19$ & $19 \sim 30$ & $30 \sim 45$ & $>45$ \\
\hline IBS & & & & & & & \\
Excellent grade & & 0.65 & 0.65 & 0.60 & 0.55 & 0.55 & 0.50 \\
First grade & Mpa & 0.60 & 0.60 & 0.55 & 0.50 & 0.50 & 0.45 & 0.50 \\
Accepted product & & 0.55 & 0.55 & 0.50 & 0.45 & 0.45 & 0.45 & 0.45 \\
\hline MOR & Mpa & 23 & 23 & 22 & 20 & 18 & 17 \\
\hline MOE & Mpa & 2700 & 2700 & 2500 & 2200 & 2100 & 1900 \\
\hline
\end{tabular}

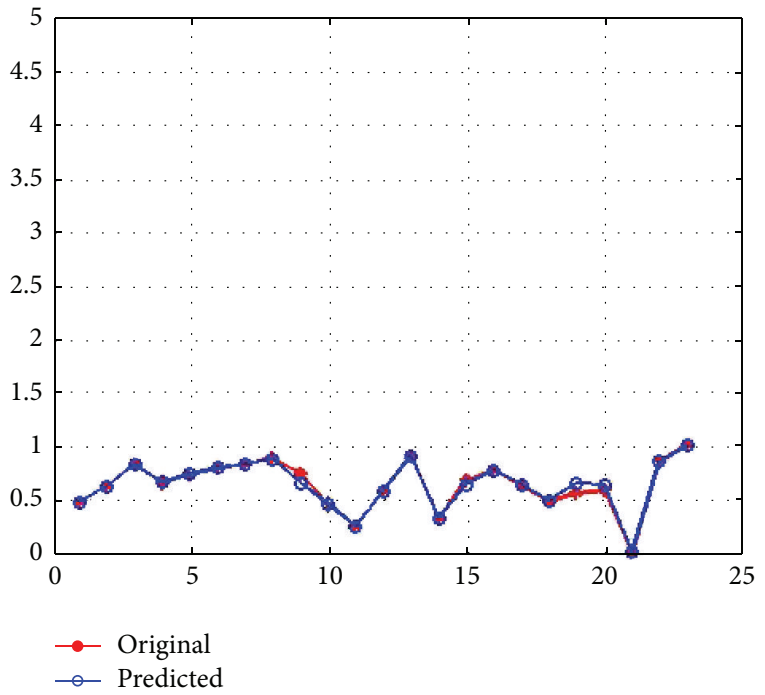

FIGURE 2: Normalized sample fitting results of network (34) for the regression of MOR.

Moreover, the regression functions based on the linear polynomial kernel in (20) are also calculated by network (34), where the parameters are also shown in Table 5.

\subsection{Optimization of Parameters in MDF Continuous Hot-} Pressing Process. In this subsection, we consider two classes of parameter optimizing problems, one aims at optimizing one performance index of MDF, and the other is for comprehensively optimizing the three performance indexes of MDF. Based on the obtained relation models for a particular slab and hot-pressing system, we give a suggestion on the setting of hot-pressing pressure, hot-pressing temperature, hot-pressing time, and moisture content of slab to let the MDF meet the given requirements. We refer to the current standard of MDF indoor plate in China (GB/T11718-1999), which is given in Table 2 .

\subsubsection{Case 1}

Case 1. When the MOR and MOE of the hot-pressing plate are in the certain regions, we would like to maximize the IBS by control of the hot-pressing pressure, hot-pressing temperature, hot-pressing time, and moisture content of slab.

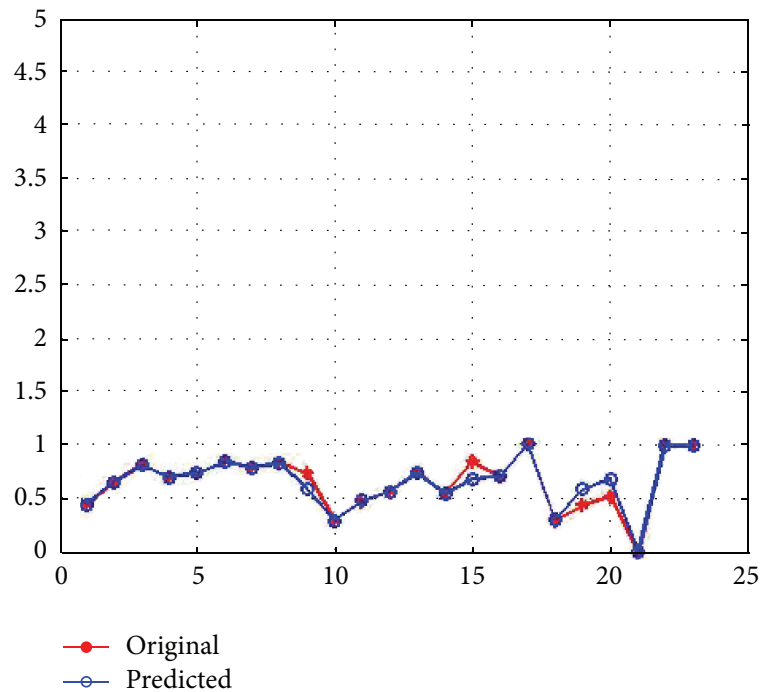

FIGURE 3: Normalized sample fitting results of network (34) for the regression of MOE.

By the information in Table 2, we let $v_{1}=200, v_{2}=4$, $\nu_{3}=7$, and $\nu_{4}=12 \%$ and choose $y_{1}^{*}=22, y_{2}^{*}=2500, \iota_{1}=8$, $\varepsilon_{1}=8, \iota_{2}=500$, and $\varepsilon_{2}=500$ in optimization problem (21).

Then, $\Omega=\left\{x \in R^{4}: 0 \leq x_{1} \leq v_{1}, 0 \leq x_{2} \leq v_{2}, 0 \leq x_{3} \leq\right.$ $\left.v_{3}, 0 \leq x_{4} \leq v_{4}\right\}$, and we can let

$$
\begin{aligned}
& g_{1}(x)=-\sum_{i=1}^{n} \bar{\rho}_{i}^{*}\left\langle z_{i}, x\right\rangle-\bar{b}_{1}+y_{1}^{*}-\iota_{1}, \\
& g_{2}(x)=\sum_{i=1}^{n} \bar{\rho}_{i}^{*}\left\langle z_{i}, x\right\rangle+\bar{b}_{1}-y_{1}^{*}-\varepsilon_{1}, \\
& g_{3}(x)=-\sum_{i=1}^{n} \bar{\theta}_{i}^{*}\left\langle z_{i}, x\right\rangle-\bar{b}_{2}+y_{2}^{*}-\iota_{2}, \\
& g_{4}(x)=\sum_{i=1}^{n} \bar{\theta}_{i}^{*}\left\langle z_{i}, x\right\rangle+\bar{b}_{2}-y_{2}^{*}-\varepsilon_{2} .
\end{aligned}
$$

By the proposed network (34) for solving (21), we obtain the optimal solution

$$
x^{*}=(159.4725,3.1022,6.4866,6.0061)^{T} .
$$




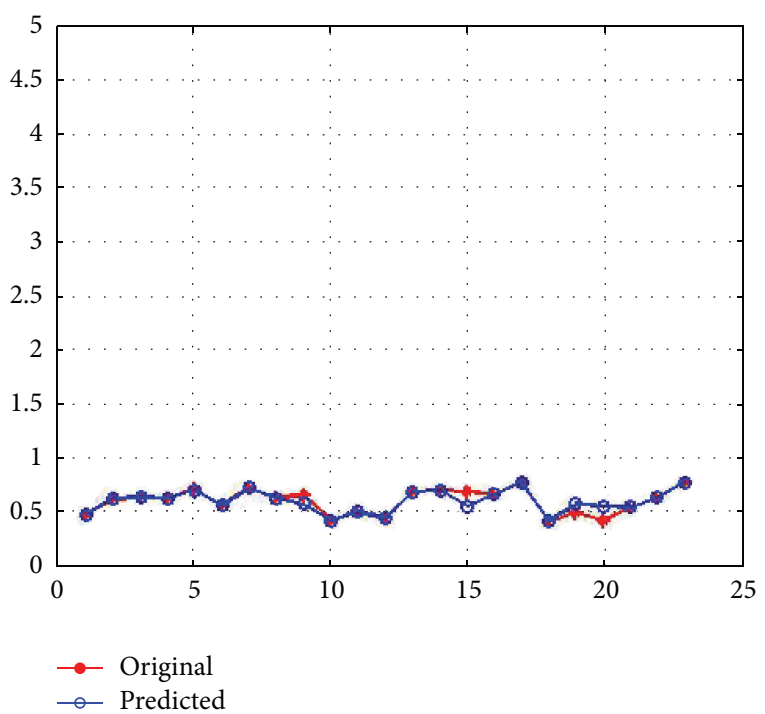

FIgURE 4: Normalized sample fitting results of network (34) for the regression of IBS.

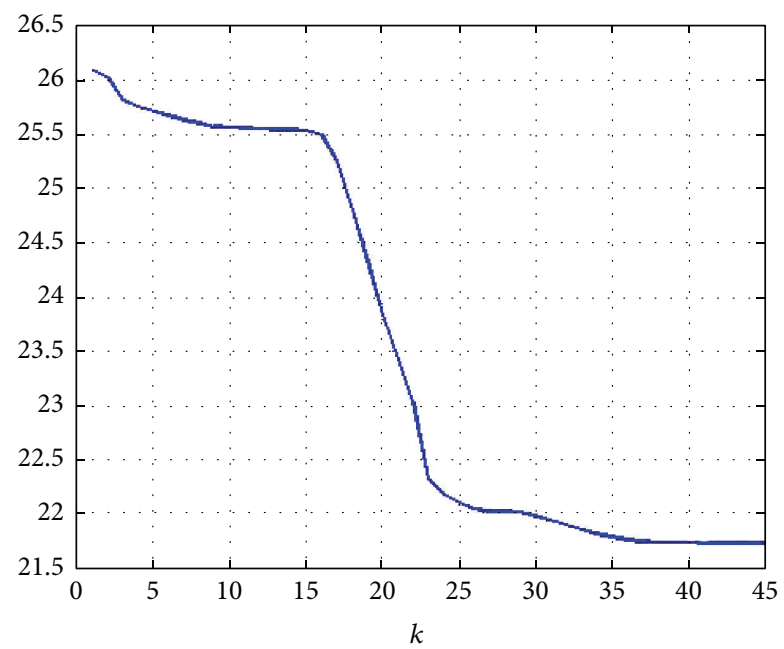

FIGURE 5: Convergence of MOR regression function $f_{1}(x)$ along the solution of (34).

TABLE 3: Case 1: performance index values of obtained hot-pressing plate.

\begin{tabular}{lcc}
\hline MOR (Mpa) & MOE $(\mathrm{Mpa})$ & IBS \\
\hline 21.7247 & 2650.4081 & 0.6040 \\
\hline
\end{tabular}

This means that when we let the hot-pressing temperature be $159.4725^{\circ} \mathrm{C}$, hot-pressing pressure be $3.1022 \mathrm{Mpa}$, hotpressing time be $6.4866 \mathrm{~min}$, and moisture content of slab be $6.0061 \%$, we can maximize the IBS of the hot-pressing plate and let the MOR and MOE of it be in the certain regions, where the three performance indexes are shown in Table 3. The convergence of the MOR regression function $f_{1}(x), \mathrm{MOE}$ regression function $f_{2}(x)$, and IBS regression function $f_{3}(x)$ along the solution of (34) are plotted in Figures 5-7.

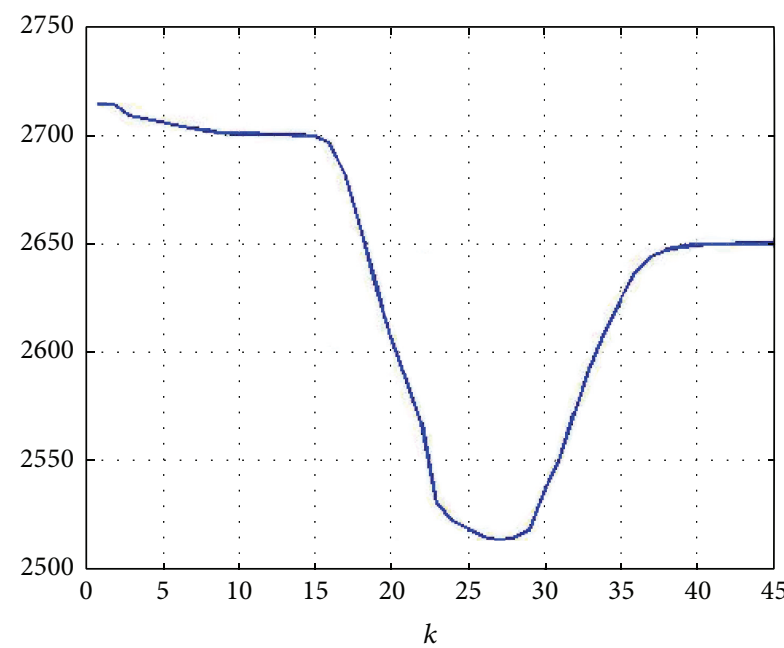

FIGURE 6: Convergence of MOE regression function $f_{2}(x)$ along the solution of (34).

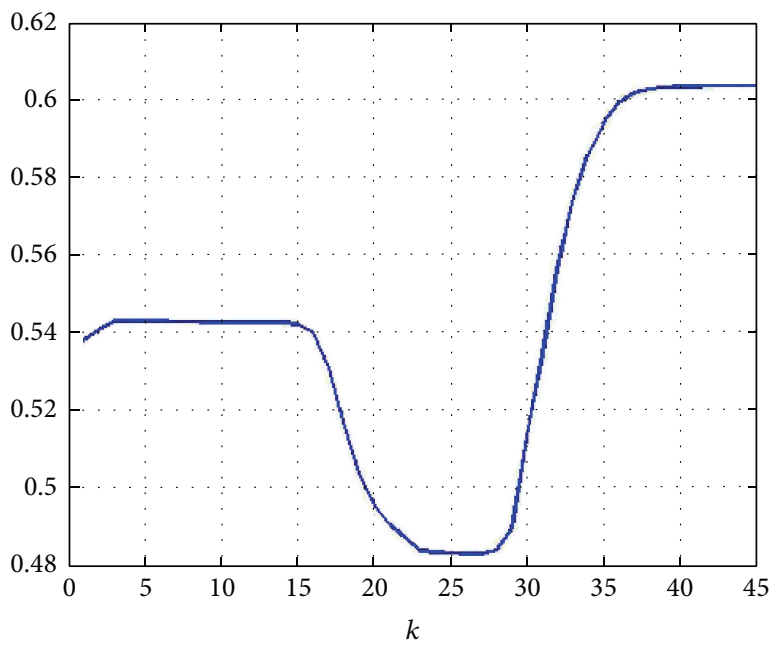

FIGURE 7: Convergence of IBS regression function $f_{3}(x)$ along the solution of (34).

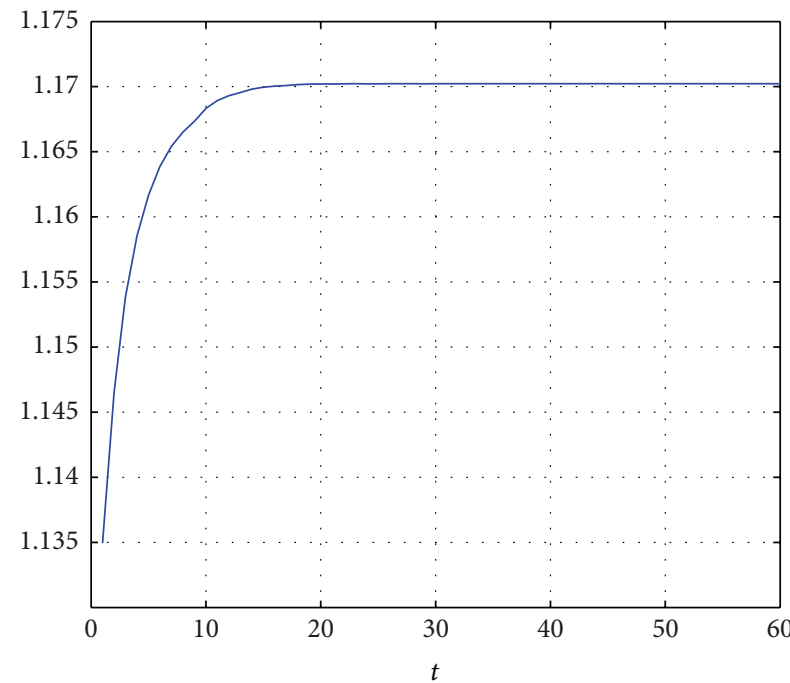

Figure 8: Convergence of $f(x)$ along the solution of (34). 
TABLE 4: Experimental data set in MDF hot-pressing.

\begin{tabular}{|c|c|c|c|c|c|c|c|}
\hline Number & $\mathrm{TE}\left({ }^{\circ} \mathrm{C}\right)$ & $\mathrm{PR}(\mathrm{MPa})$ & $\mathrm{TI}(\mathrm{min})$ & MC (\%) & MOR (Mpa) & MOE (MPa) & IB (Mpa) \\
\hline 1 & 170 & 3 & 4 & 8 & 25.66 & 2538.93 & 0.4775 \\
\hline 2 & 170 & 3.4 & 5 & 10 & 29.1275 & 2887.788 & 0.62 \\
\hline 3 & 170 & 2.7 & 6 & 12 & 33.95 & 3159.645 & 0.635 \\
\hline 4 & 180 & 3 & 6 & 10 & 29.9025 & 2967.803 & 0.625 \\
\hline 5 & 180 & 3.4 & 4 & 12 & 31.76 & 3034.485 & 0.7025 \\
\hline 6 & 180 & 2.7 & 5 & 8 & 33.1925 & 3215.603 & 0.5625 \\
\hline 7 & 190 & 3 & 5 & 12 & 33.98 & 3122.16 & 0.72 \\
\hline 8 & 190 & 3.4 & 6 & 8 & 35.1575 & 3191.608 & 0.63 \\
\hline 9 & 190 & 2.7 & 4 & 10 & 31.955 & 3034.818 & 0.65 \\
\hline 10 & 180 & 3 & 4 & 8 & 25.13 & 2296.26 & 0.42 \\
\hline 11 & 170 & 3.4 & 4 & 10 & 20.36 & 2601.56 & 0.5 \\
\hline 12 & 170 & 2.7 & 4 & 12 & 28 & 2743.05 & 0.44 \\
\hline 13 & 190 & 3 & 4 & 12 & 35.74 & 3063.48 & 0.687 \\
\hline 14 & 180 & 3.4 & 4 & 8 & 22.2025 & 2707.743 & 0.7 \\
\hline 15 & 190 & 2.7 & 5 & 10 & 30.85 & 3212.7 & 0.68 \\
\hline 16 & 190 & 3 & 6 & 12 & 32.65 & 2982.33 & 0.66 \\
\hline 17 & 170 & 3.4 & 6 & 10 & 29.38 & 3487.39 & 0.77 \\
\hline 18 & 180 & 2.7 & 4 & 8 & 25.87 & 2304.19 & 0.41 \\
\hline 19 & 190 & 2.7 & 6 & 10 & 27.63 & 2539.05 & 0.49 \\
\hline 20 & 180 & 2.7 & 5 & 10 & 28.35 & 2675.37 & 0.42 \\
\hline 21 & 170 & 2.7 & 5 & 8 & 14.63 & 1806.99 & 0.5431 \\
\hline 22 & 180 & 3 & 5 & 12 & 34.52 & 3462.12 & 0.63 \\
\hline 23 & 190 & 3.4 & 5 & 10 & 38.07 & 3457.79 & 0.77 \\
\hline
\end{tabular}

TABLE 5: Parameter values in the regression functions.

\begin{tabular}{|c|c|c|c|c|c|c|c|c|c|c|c|}
\hline \multicolumn{6}{|c|}{ In the regression functions (19) } & & \multicolumn{5}{|c|}{ In the regression functions (20) } \\
\hline$\overline{\rho_{1}^{*}}$ & -0.1385 & $\theta_{1}^{*}$ & -0.0335 & $\kappa_{1}^{*}$ & -0.073 & $\bar{\rho}_{1}^{*}$ & 2.5815 & $\bar{\theta}_{1}^{*}$ & 1.37581 & $\bar{\kappa}_{1}^{*}$ & -0.5275 \\
\hline$\rho_{2}^{*}$ & 0.0139 & $\theta_{2}^{*}$ & -0.0794 & $\kappa_{2}^{*}$ & -0.0060 & $\bar{\rho}_{2}^{*}$ & 1.1358 & $\bar{\theta}_{2}^{*}$ & -0.7298 & $\bar{\kappa}_{2}^{*}$ & -6.5132 \\
\hline$\rho_{3}^{*}$ & 0.1869 & $\theta_{3}^{*}$ & 0.1332 & $\kappa_{3}^{*}$ & 0.0259 & $\bar{\rho}_{3}^{*}$ & 2.4638 & $\bar{\theta}_{3}^{*}$ & 1.0424 & $\bar{\kappa}_{3}^{*}$ & 9.7535 \\
\hline$\rho_{4}^{*}$ & 0.0119 & $\theta_{4}^{*}$ & -0.0365 & $\kappa_{4}^{*}$ & -0.0000 & $\bar{\rho}_{4}^{*}$ & -1.0246 & $\bar{\theta}_{4}^{*}$ & -1.3607 & $\bar{\kappa}_{4}^{*}$ & -5.9500 \\
\hline$\rho_{5}^{*}$ & 0.0948 & $\theta_{5}^{*}$ & 0.0554 & $\kappa_{5}^{*}$ & 0.0939 & $\bar{\rho}_{5}^{*}$ & 0.1880 & $\bar{\theta}_{5}^{*}$ & -0.6708 & $\bar{\kappa}_{5}^{*}$ & 6.3092 \\
\hline$\rho_{6}^{*}$ & 0.2217 & $\theta_{6}^{*}$ & 0.5229 & $\kappa_{6}^{*}$ & 0.0218 & $\bar{\rho}_{6}^{*}$ & 4.8889 & $\bar{\theta}_{6}^{*}$ & 6.1594 & $\bar{\kappa}_{6}^{*}$ & 15.5774 \\
\hline$\rho_{7}^{*}$ & 0.1409 & $\theta_{7}^{*}$ & 0.0001 & $\kappa_{7}^{*}$ & 0.0823 & $\bar{\rho}_{7}^{*}$ & -0.9675 & $\bar{\theta}_{7}^{*}$ & -1.2267 & $\bar{\kappa}_{7}^{*}$ & 10.3006 \\
\hline$\rho_{8}^{*}$ & 0.2361 & $\theta_{8}^{*}$ & 0.1338 & $\kappa_{8}^{*}$ & 0.0054 & $\bar{\rho}_{8}^{*}$ & 1.6596 & $\bar{\theta}_{8}^{*}$ & -0.1886 & $\bar{\kappa}_{8}^{*}$ & -23.9383 \\
\hline$\rho_{9}^{*}$ & 50.0000 & $\theta_{9}^{*}$ & 50.0000 & $\kappa_{9}^{*}$ & 45.8063 & $\bar{\rho}_{9}^{*}$ & 1.2497 & $\bar{\theta}_{9}^{*}$ & 3.0084 & $\bar{\kappa}_{9}^{*}$ & 30.8681 \\
\hline$\rho_{10}^{*}$ & -0.1413 & $\theta_{10}^{*}$ & -0.2405 & $\kappa_{10}^{*}$ & -0.1362 & $\bar{\rho}_{10}^{*}$ & 0.0644 & $\bar{\theta}_{10}^{*}$ & -2.2099 & $\bar{\kappa}_{10}^{*}$ & -25.9216 \\
\hline$\rho_{11}^{*}$ & -0.3874 & $\theta_{11}^{*}$ & -0.1625 & $\kappa_{11}^{*}$ & -0.1190 & $\bar{\rho}_{11}^{*}$ & 3.6153 & $\bar{\theta}_{11}^{*}$ & -1.6600 & $\bar{\kappa}_{11}^{*}$ & -27.0340 \\
\hline$\rho_{12}^{*}$ & -0.0627 & $\theta_{12}^{*}$ & -0.1128 & $\kappa_{12}^{*}$ & -0.1673 & $\bar{\rho}_{12}^{*}$ & -0.8880 & $\bar{\theta}_{12}^{*}$ & 0.7098 & $\bar{\kappa}_{12}^{*}$ & -17.8497 \\
\hline$\rho_{13}^{*}$ & 0.2501 & $\theta_{13}^{*}$ & 0.0338 & $\kappa_{13}^{*}$ & 0.0488 & $\bar{\rho}_{13}^{*}$ & 1.4724 & $\bar{\theta}_{13}^{*}$ & -0.1911 & $\bar{\kappa}_{13}^{*}$ & 13.6398 \\
\hline$\rho_{14}^{*}$ & -0.3013 & $\theta_{14}^{*}$ & -0.0673 & $\kappa_{14}^{*}$ & 0.1250 & $\bar{\rho}_{14}^{*}$ & -2.5732 & $\bar{\theta}_{14}^{*}$ & 0.0263 & $\bar{\kappa}_{14}^{*}$ & 28.5659 \\
\hline$\rho_{15}^{*}$ & 43.4098 & $\theta_{15}^{*}$ & 50.0000 & $\kappa_{15}^{*}$ & 50.0000 & $\bar{\rho}_{15}^{*}$ & -0.9224 & $\bar{\theta}_{15}^{*}$ & 2.8766 & $\bar{\kappa}_{15}^{*}$ & 26.7062 \\
\hline$\rho_{16}^{*}$ & 0.1186 & $\theta_{16}^{*}$ & 0.0058 & $\kappa_{16}^{*}$ & 0.0221 & $\bar{\rho}_{16}^{*}$ & -3.1735 & $\bar{\theta}_{16}^{*}$ & -4.4727 & $\bar{\kappa}_{16}^{*}$ & -17.4470 \\
\hline$\rho_{17}^{*}$ & -0.0048 & $\theta_{17}^{*}$ & 0.3582 & $\kappa_{17}^{*}$ & 0.1597 & $\bar{\rho}_{17}^{*}$ & 0.0304 & $\bar{\theta}_{-*}^{*}$ & 3.2063 & $\bar{\kappa}_{17}^{*}$ & 21.1381 \\
\hline$\rho_{18}^{*}$ & -0.1428 & $\theta_{18}^{*}$ & -0.3514 & $\kappa_{18}^{*}$ & -0.1356 & $\bar{\rho}_{18}^{*}$ & 1.0775 & $\bar{\theta}_{18}^{*}$ & -0.8331 & $\bar{\kappa}_{18}^{*}$ & -12.7596 \\
\hline$\rho_{19}^{*}$ & -50.0000 & $\theta_{19}^{*}$ & -50.0000 & $\kappa_{19}^{*}$ & -45.8399 & $\bar{\rho}_{19}^{*}$ & -1.6929 & $\bar{\theta}_{19}^{*}$ & -1.7854 & $\bar{\kappa}_{19}^{*}$ & -11.9152 \\
\hline$\rho_{20}^{*}$ & -43.4157 & $\theta_{20}^{*}$ & -50.0000 & $\kappa_{20}^{*}$ & -50.0000 & $\bar{\rho}_{20}^{*}$ & -2.4732 & $\bar{\theta}_{20}^{*}$ & -2.3246 & $\bar{\kappa}_{20}^{*}$ & -43.5023 \\
\hline$\rho_{21}^{*}$ & -0.6476 & $\theta_{21}^{*}$ & -0.7696 & $\kappa_{21}^{*}$ & -0.0472 & $\bar{\rho}_{21}^{*}$ & -05.8057 & $\bar{\theta}_{21}^{*}$ & -6.3103 & $\bar{\kappa}_{21}^{*}$ & 19.8815 \\
\hline$\rho_{22}^{*}$ & 0.1968 & $\theta_{22}^{*}$ & -0.3108 & $\kappa_{22}^{*}$ & -0.0101 & $\bar{\rho}_{22}^{*}$ & 1.4923 & $\bar{\theta}_{22}^{*}$ & 3.3107 & $\bar{\kappa}_{22}^{*}$ & -3.6604 \\
\hline$\rho_{23}^{*}$ & 0.3606 & $\theta_{23}^{*}$ & 0.3021 & $\kappa_{23}^{*}$ & 0.1471 & $\bar{\rho}_{23}^{*}$ & 3.0559 & $\bar{\theta}_{23}^{*}$ & 2.2478 & $\bar{\kappa}_{23}^{*}$ & 14.2783 \\
\hline$b_{1}$ & 0.6361 & $b_{2}$ & 0.6554 & $b_{3}$ & 0.6052 & $\bar{b}_{1}$ & 0.2784 & $\bar{b}_{2}$ & 0.2693 & $\bar{b}_{3}$ & 0.4230 \\
\hline
\end{tabular}




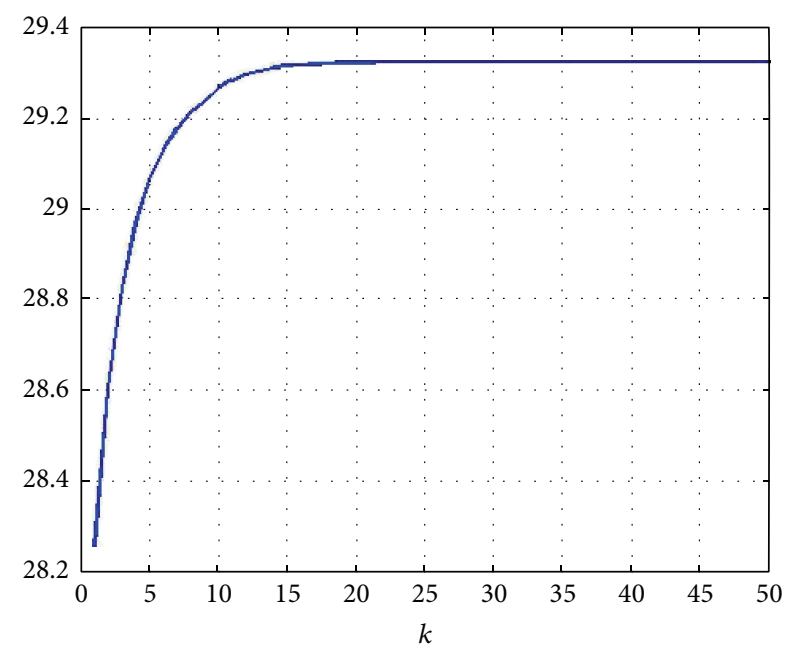

FIGURE 9: Convergence of MOR regression function $f_{1}(x)$ along the solution of (34).

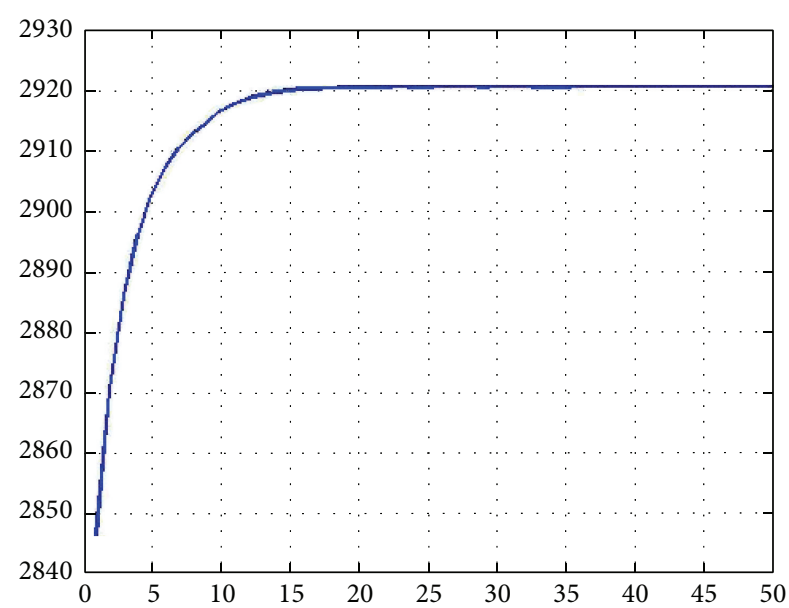

FIGURE 10: Convergence of MOE regression function $f_{2}(x)$ along the solution of (34).

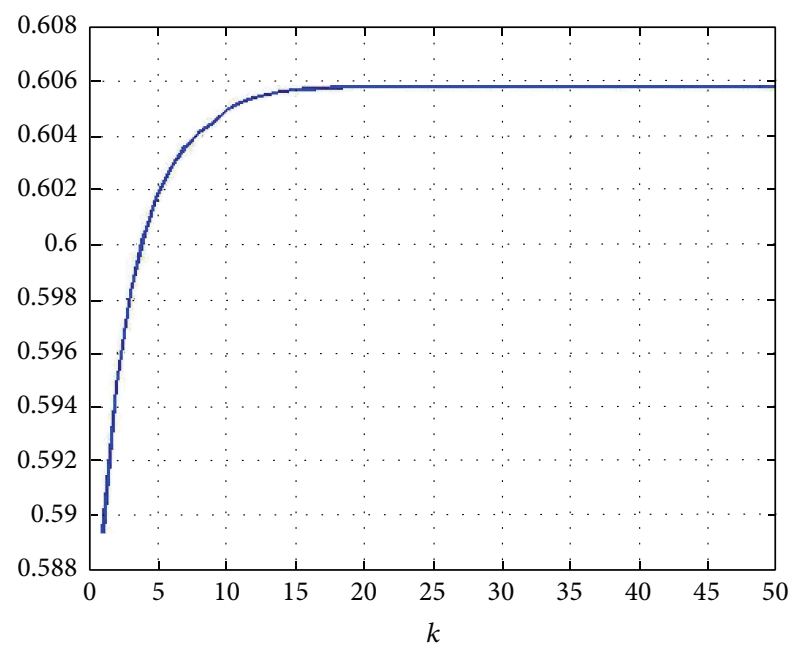

FIGURE 11: Convergence of IBS regression function $f_{3}(x)$ along the solution of (34).

\subsubsection{Case 2}

Case 2. We would like to maximize the MOR, MOE, and IBS synthetically by control of the hot-pressing pressure, hot-pressing temperature, hot-pressing time, and moisture content of slab.

In this case, we use optimization problem (23) with $\lambda_{1}=$ $1 / 3, \lambda_{2}=1 / 3, \lambda_{3}=1 / 3, v_{1}=200, v_{2}=4, v_{3}=7, v_{4}=12 \%$, $f_{1}^{*}=22(\mathrm{Mpa}), f_{2}^{*}=2500(\mathrm{Mpa})$, and $f_{3}^{*}=0.60(\mathrm{Mpa})$.

Using proposed network (34) to solve (23), we obtain the optimal solution

$$
x^{*}=(182.2461,3.0991,8.5167,5.1801)^{T} .
$$

The convergence of $f(x)$ along the solution of (23) is plotted in Figure 8 , where the convergence of MOR regression function $f_{1}(x)$, MOE regression function $f_{2}(x)$, and IBS regression function $f_{3}(x)$ is shown in Figures 9-11.

\section{Competing Interests}

The authors declare that they have no competing interests.

\section{Acknowledgments}

This work is supported by the Fundamental Research Funds for the Central Universities (2572014AB03) and the National Natural Science Foundation of China (31370565).

\section{References}

[1] M. Irle and C. Loxton, "The manufacture and use of panel products in the UK," Journal of the Institute of Wood Science, vol. 14, no. 1, pp. 21-26, 1996.

[2] D. Olah, R. Smith, and B. Hansen, "Wood material use in the U.S. cabinet industry 1999 to 2001," Forest Products Journal, vol. 53, no. 1, pp. 25-31, 2003.

[3] J. J. Hopfield and D. W. Tank, "Neural' computation of decisons in optimization problems," Biological Cybernetics, vol. 52, no. 3, pp. 141-152, 1985.

[4] M. P. Kennedy and L. O. Chua, "Neural networks for nonlinear programming," IEEE Transactions on Circuits and Systems, vol. 35, no. 5, pp. 554-562, 1988.

[5] D. W. Tank and J. J. Hopfield, "Simple 'neural' optimization networks: an A/D converter, signal decision circuit, and a linear programming circuit," IEEE Transactions on Circuits and Systems, vol. 33, no. 5, pp. 533-541, 1986.

[6] W. Bian and X. P. Xue, "Subgradient-based neural networks for nonsmooth nonconvex optimization problems," IEEE Transactions on Neural Networks, vol. 20, pp. 1024-1038, 2009.

[7] W. Bian and X. Xue, "Neural network for solving constrained convex optimization problems with global attractivity," IEEE Transactions on Circuits and Systems. I. Regular Papers, vol. 60, no. 3, pp. 710-723, 2013.

[8] L. Cheng, Z.-G. Hou, Y. Z. Lin, M. Tan, W. C. Zhang, and F.-X. Wu, "Recurrent neural network for non-smooth convex optimization problems with application to the identification of genetic regulatory networks," IEEE Transactions on Neural Networks, vol. 22, no. 5, pp. 714-726, 2011. 
[9] Q. Liu, Z. Guo, and J. Wang, "A one-layer recurrent neural network for constrained pseudoconvex optimization and its application for dynamic portfolio optimization," Neural Networks, vol. 26, pp. 99-109, 2012.

[10] S. Zhang and A. G. Constantinides, "Lagrange programming neural networks," IEEE Transactions on Circuits and Systems II: Analog and Digital Signal Processing, vol. 39, no. 7, pp. 441-452, 1992.

[11] X. Hu and J. Wang, "Design of general projection neural networks for solving monotone linear variational inequalities and linear and quadratic optimization problems," IEEE Transactions on Systems, Man, and Cybernetics Part B: Cybernetics, vol. 37, no. 5, pp. 1414-1421, 2007.

[12] Q. Liu and J. Cao, "A recurrent neural network based on projection operator for extended general variational inequalities," IEEE Transactions on Systems, Man, and Cybernetics, Part B: Cybernetics, vol. 40, no. 3, pp. 928-938, 2010.

[13] Y. Xia, G. Feng, and J. Wang, "A recurrent neural network with exponential convergence for solving convex quadratic program and related linear piecewise equations," Neural Networks, vol. 17, no. 7, pp. 1003-1015, 2004.

[14] Q. X. Zhu and J. D. Cao, "Exponential stability of stochastic neural networks with both Markovian jump parameters and mixed time delays," IEEE Transactions on Systems, Man, and Cybernetics, Part B: Cybernetics, vol. 41, no. 2, pp. 341-353, 2011.

[15] M. Forti, P. Nistri, and M. Quincampoix, "Generalized neural network for nonsmooth nonlinear programming problems," IEEE Transactions on Circuits and Systems I: Regular Papers, vol. 51, no. 9, pp. 1741-1754, 2004.

[16] M. Forti, P. Nistri, and M. Quincampoix, "Convergence of neural networks for programming problems via a nonsmooth Łojasiewicz inequality," IEEE Transactions on Neural Networks, vol. 17, no. 6, pp. 1471-1486, 2006.

[17] W. L. Lu and J. Wang, "Convergence analysis of a class of nonsmooth gradient systems," IEEE Transactions on Circuits and Systems I: Regular Papers, vol. 55, no. 11, pp. 3514-3527, 2008.

[18] W. Bian and X. Chen, "Smoothing neural network for constrained non-lipschitz optimization with applications," IEEE Transactions on Neural Networks and Learning Systems, vol. 23, no. 3, pp. 399-411, 2012.

[19] F. H. Clarke, Optimization and Nonsmooth Analysis, John Wiley \& Sons, New York, NY, USA, 1983.

[20] R. T. Rockafellar and R. J. Wets, Variational Analysis, vol. 317 of Grundlehren der Mathematischen Wissenschaften, Springer, Berline, Germany, 1998.

[21] B. Scholkopf and A. J. Smola, Learning with Kernels, MIT Press, Cambridge, Mass, USA, 2002.

[22] F. Liu and X. Xue, "Design of natural classification kernels using prior knowledge," IEEE Transactions on Fuzzy Systems, vol. 20, no. 1, pp. 135-152, 2012.

[23] X. Chen, "Smoothing methods for nonsmooth, nonconvex minimization," Mathematical Programming, vol. 134, no. 1, pp. 71-99, 2012.

[24] C. Chen and O. L. Mangasarian, "A class of smoothing functions for nonlinear and mixed complementarity problems," Computational Optimization and Applications, vol. 5, no. 2, pp. 97-138, 1996.

[25] J.-P. Penot and P. H. Quang, "Generalized convexity of functions and generalized monotonicity of set-valued maps," Journal of Optimization Theory and Applications, vol. 92, no. 2, pp. 343356, 1997. 


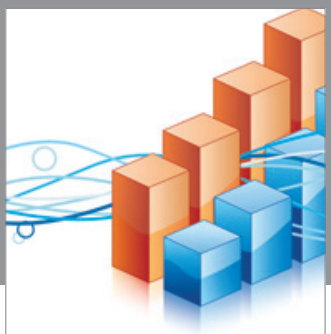

Advances in

Operations Research

vatem alat4

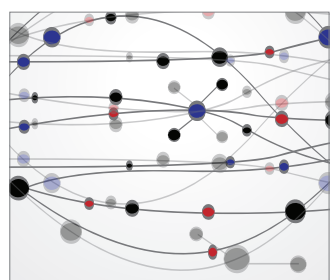

\section{The Scientific} World Journal
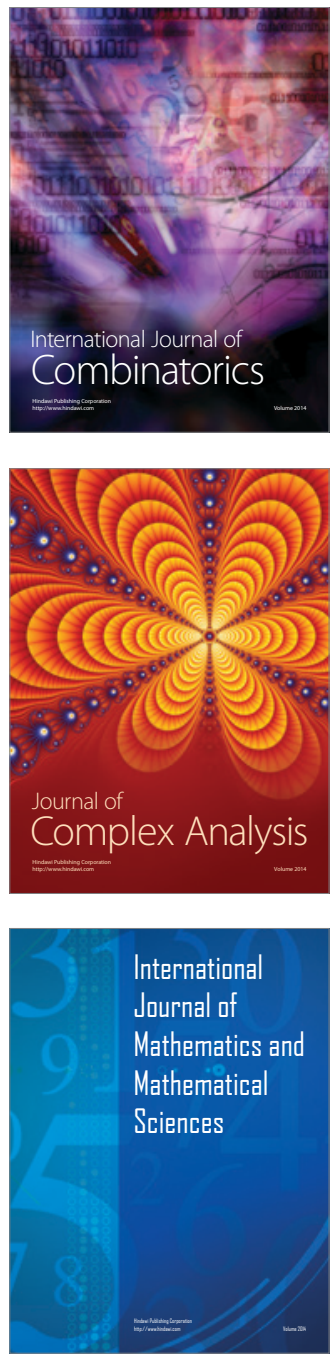
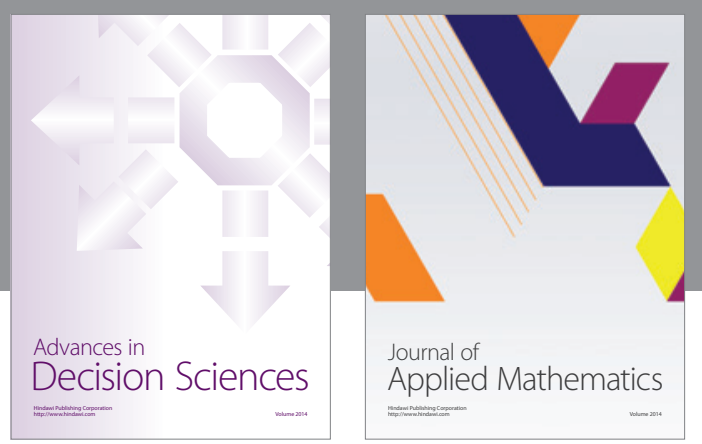

Algebra

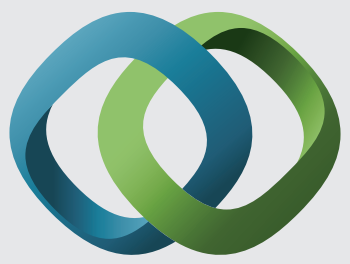

\section{Hindawi}

Submit your manuscripts at

http://www.hindawi.com
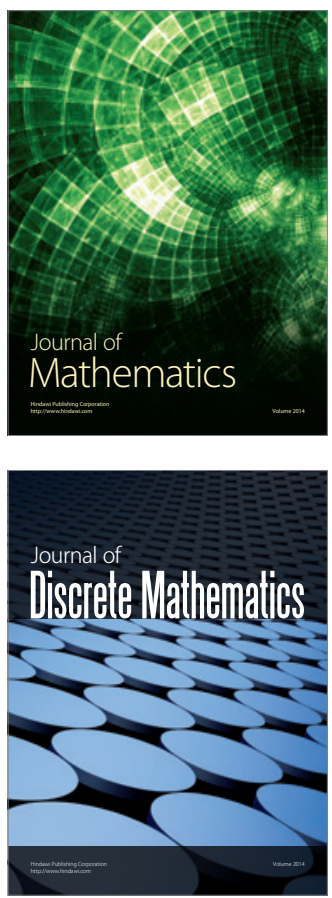

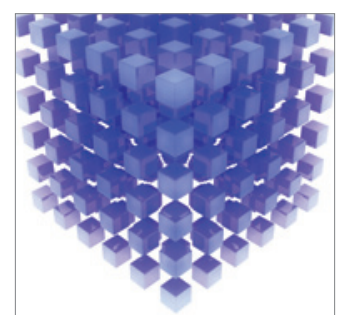

Mathematical Problems in Engineering
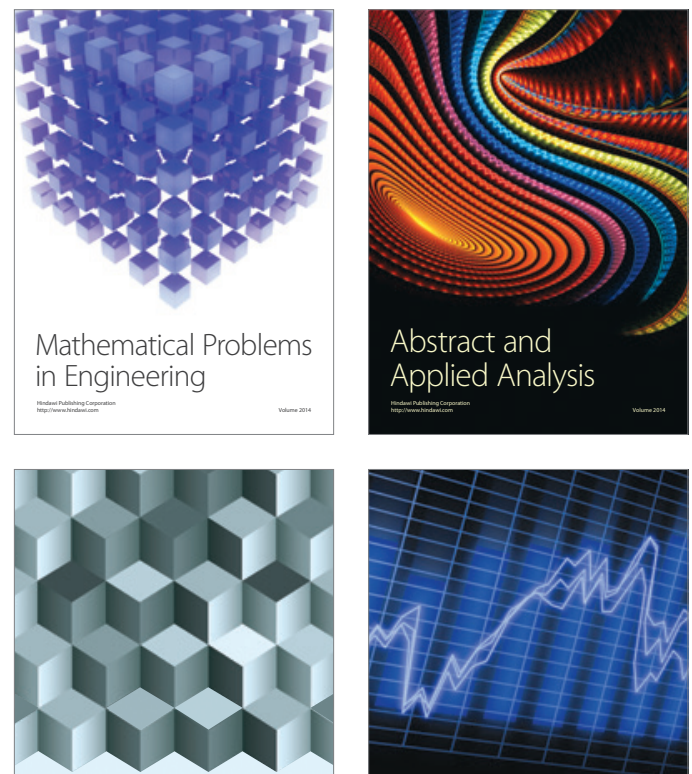

Journal of

Function Spaces

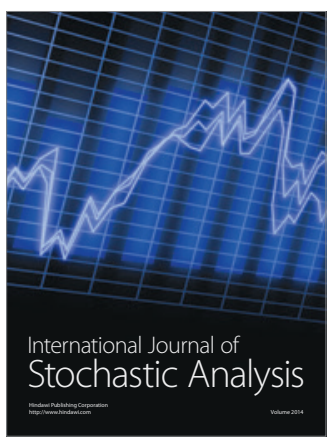

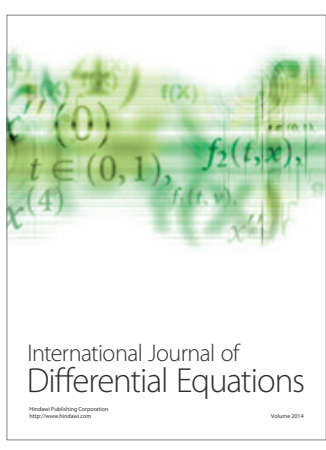
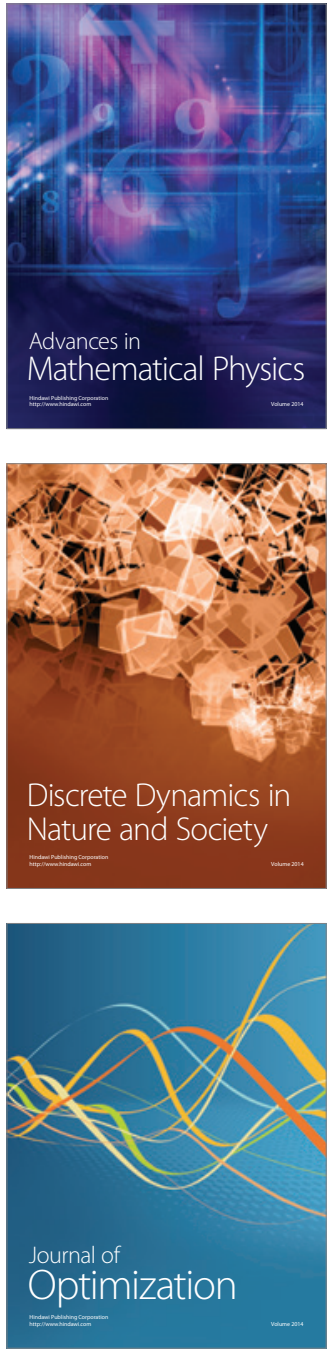